\title{
Naturally-Occurring Rare Mutations Cause Mild to Catastrophic Effects in the Multifunctional and Cancer-Associated NQO1 Protein
}

\author{
Juan Luis Pacheco-García ${ }^{1}{ }^{\circledR}$, Mario Cano-Muñoz ${ }^{1}{ }^{\circledR}$, Isabel Sánchez-Ramos ${ }^{1}$, Eduardo Salido ${ }^{2}$ \\ and Angel L. Pey ${ }^{3, * \mathbb{D}}$ \\ 1 Departamento de Química Física, Facultad de Ciencias, Universidad de Granada, 18071 Granada, Spain; \\ juanlupacheco@correo.ugr.es (J.L.P.-G.); mariocano@ugr.es (M.C.-M.); isanchezb@correo.ugr.es (I.S.-R.) \\ 2 Centre for Biomedical Research on Rare Diseases (CIBERER), Hospital Universitario de Canarias, \\ 38320 Tenerife, Spain; edsalido@gmail.com \\ 3 Departamento de Química Física y Unidad de Excelencia de Química Aplicada a Biomedicina y \\ Medioambiente (UEQ), Facultad de Ciencias, Universidad de Granada, 18071 Granada, Spain \\ * Correspondence: angelpey@ugr.es; Tel.: +34-958243173
}

Received: 7 October 2020; Accepted: 2 November 2020; Published: 3 November 2020

\begin{abstract}
The functional and pathological implications of the enormous genetic diversity of the human genome are mostly unknown, primarily due to our unability to predict pathogenicity in a high-throughput manner. In this work, we characterized the phenotypic consequences of eight naturally-occurring missense variants on the multifunctional and disease-associated NQO1 protein using biophysical and structural analyses on several protein traits. Mutations found in both exome-sequencing initiatives and in cancer cell lines cause mild to catastrophic effects on NQO1 stability and function. Importantly, some mutations perturb functional features located structurally far from the mutated site. These effects are well rationalized by considering the nature of the mutation, its location in protein structure and the local stability of its environment. Using a set of 22 experimentally characterized mutations in NQO1, we generated experimental scores for pathogenicity that correlate reasonably well with bioinformatic scores derived from a set of commonly used algorithms, although the latter fail to semiquantitatively predict the phenotypic alterations caused by a significant fraction of mutations individually. These results provide insight into the propagation of mutational effects on multifunctional proteins, the implementation of in silico approaches for establishing genotype-phenotype correlations and the molecular determinants underlying loss-of-function in genetic diseases.
\end{abstract}

Keywords: missense mutation; genetic disease; protein structure-function; genotype-phenotype correlations; multifunctional proteins

\section{Introduction}

Due to technological advances in DNA sequencing technology, we are begining to realize the tremendous genetic variability in the human genome [1,2] and to accurately map the genetic alterations associated with certain diseases [3]. A single human genome carries thousands of missense mutations $[1,2,4]$. However, the potential physio-pathological implications of this genetic diversity are unclear, partly due to our limited ability to predict the effects of missense variants on predisposition to disease [5,6]. Our limited capacity for establishing large-scale genotype-phenotype correlations can be explained by different reasons: (i) the relationships between molecular, pathogenic and organismal effects are complex, even for diseases with simple Mendelian inheritance $[1,7,8]$; (ii) genotype-phenotype correlations are improved when experimental data for molecular effects of 
mutations are available, but current predictive tools still underperform experimental characterization, particularly for mild to moderate phenotypes [8-11]; (iii) although many diseases are caused by loss-of-function mutations [12], an operational definition of loss-of-function is difficult because human proteins display many functional and regulatory traits. In this context, genotype-phenotype correlations require an integrated understanding on how a single mutation may affect multiple molecular functions simultaneously and, consequently, how these molecular effects translate into pathogenic and fitness consequences [1,13-23].

The molecular mechanisms by which missense mutations cause loss-of-function are many-fold [24] and include accelerated protein degradation $[5,9,11,25,26]$, enhanced protein aggregation $[14,15,21]$, catalytic and regulatory alterations [21,27-29] and altered biomacromolecular interactions $[10,30]$. Importantly, the common molecular origin for all these coexisting mechanisms appears to be the structural and energetic perturbation caused by missense mutations $[5,8,11,19,24,26]$. These perturbations may differently contribute to the molecular phenotype associated with a given missense variant [19]. Therefore, a fundamental issue to understand the effect of missense variants on multifunctional proteins will come from a deep knowledge on how mutational effects are propagated through the protein structure affecting multiple functional features [19,31].

There are several strategies to develop tools for estimating the impact of missense mutations on protein stability and establishing the potential pathogenicity of missense variants that can be broadly classified into: (i) sequence-based methods that use multiple sequence analysis combined with simple physical estimations of the mutational effect [5,32] — a clear advantage of these methods is that high-resolution structural information on the protein of interest is not required; (ii) structure-based methods that (quantitatively) evaluate mutational effects on protein thermodynamic stability (as a change in the unfolding free energy, $\Delta \mathrm{G}_{\mathrm{U}}$ ). These are often trained and tested using large datasets of experimentally determined mutational effects on $\Delta \mathrm{G}_{\mathrm{U}}$ [33-36] and work rather well overall but fail for individual mutations [37]. Importantly, mutational effects are universally distributed across protein structures, with solvent-exposed mutations causing much milder effects than those affecting buried residues $[35,36]$. These studies have three important implications to understand the functional consequences of the vast genetic diversity present in the human genome. First, between $1 / 2$ and $2 / 3$ of all possible mutations have minimal effects on conformational stability $[35,36]$ and mild functional effects in vivo [38]. Second, rather small effects on conformational stability $\left(\Delta \Delta \mathrm{G}_{\mathrm{U}} \sim-2 \mathrm{kcal} \cdot \mathrm{mol}^{-1}\right)$ can subtantially accelerate protein degradation and cause loss-of-function $[5,9,11,26]$. Third, missense mutations may differently affect native vs. partially folded states and, consequently, operate through different loss-of-function mechanisms [19,39].

We have recently used the enzyme $\mathrm{NAD}(\mathrm{P}) \mathrm{H}$ quinone oxidoreductase 1 (NQO1; EC 1.6.5.2) to investigate how missense mutations may affect different functional features in a multifunctional human protein [19,39-45]. NQO1 folds into functional dimers of $62 \mathrm{kDa}[46,47]$ and each monomer has 274 residues divided into two domains: an N-terminal domain (NTD, residues 1-225) that contains a tightly bound FAD and most of the catalytic site and the monomer:monomer interface (MMI), and a C-terminal domain (CTD, residues 225-274) that completes the MMI and the active site [44,46,48-51]. FAD binding is essential for NQO1 function and intracellular stability, since the wild-type (WT) protein lacking bound cofactor ( $\mathrm{NQO1}_{\mathrm{apo}}$ ) is highly flexible and rapidly targeted for degradation [41,44,49,52]. NQO1 is a multifunctional protein with enzymatic and non-enzymatic functions [47,53]. NQO1 catalyzes the two electron reduction of quinones, acting in the antioxidant defense, maintaining several vitamers and biomolecules in their reduced state and scavenging superoxide radicals [54-58]. The enzymatic cycle contains two half-reactions following a ping-pong mechanism: a first step in which $\mathrm{NAD}(\mathrm{P}) \mathrm{H}$ binds to the FAD-bound enzyme $\left(\mathrm{NQO}_{\text {holo }}\right)$, reducing the flavin to $\mathrm{FADH}_{2}$ and releasing $\mathrm{NAD}(\mathrm{P})^{+}$, and a second half-reaction in which $\mathrm{FADH}_{2}$ reduces the bound substrate regenerating the $\mathrm{NQO}_{\text {holo }}$ state $[47,49,59]$. Dicoumarol (Dic) acts as a competitive inhibitor of $\mathrm{NAD}(\mathrm{P}) \mathrm{H}$ in the first half-reaction and also inhibitis the oxidative (second) half-reaction $[53,59,60]$. In addition, NQO1 develops key macromolecular interactions with regulatory functions, particularly protein:protein (PPI) and protein:nucleic acid 
interactions [53,58]. Particularly relevant, NQO1 drives PPI with over 20 partners, including key factors in carcinogenesis such as p53, p73 $\alpha$ and HIF- $1 \alpha$ and these interactions have chaperoning roles protecting protein partners towards intracellular degradation [53,61,62]. Furthermore, these PPIs are very sensitive to ligand binding to NQO1: reduction of FAD by $\mathrm{NAD}(\mathrm{P}) \mathrm{H}$ generally enhances the interaction, while Dic binding usually prevents it [53,62]. NQO1 seems to play a dual role in different pathological states [47]: first, NQO1 is overexpressed in cancer cells, enhancing the cellular antioxidant capacity. This has prompted research towards inhibiting NQO1 as a cancer therapy (e.g., Dic effectively inhibits cancer cells growth $[63,64])$. Second, two common single-nucleotide polymorphisms (SNPs, p.P187S and p.R139W) have been found in cancer lines and associated with increased cancer risk. The molecular consequences of these two SNPs have been characterized in some detail. P187S (c.559 C > T/p.P187S, allele frequency of $2.47 \cdot 10^{-1}$ in human population; gnomAD v.2.1.1.; https: //gnomad.broadinstitute.org/gene/ENSG00000181019?dataset=gnomad_r2_1) is strongly associated with cancer development and poor prognosis when it occurs in homocygosis [65]. This variant strongly destabilizes the protein intracellularly due to thermodynamic destabilization of the CTD that enhances its ubiquitination and acts as very efficient site for proteasomal degradation $[41,43,44,49,52,66-68]$. In addition, P187S strongly destabilizes the FAD binding site in the apo-state ( $\mathrm{NQO}_{\mathrm{apo}}$ ), decreasing by 10-40 fold the affinity for the flavin cofactor [43-45,49]. R139W (c.465 C > T/p.R139W, allele frequency of $3.11 \cdot 10^{-2}$, gnomAD v.2.1.1) is associated with increased risk of developing acute lymphoblastic leukemia in children [69]. This SNP causes minimal perturbations in the function and stability of the NQO1 protein $[40,41,70]$ but promotes aberrant RNA processing, leading to exon 4 skipping that results in an unstable and inactive NQO1 protein [71]. In addition, the consequences of a rare mutation found in a cancer cell line (COSMIC database, c.718 A > C/p.K240Q) have been characterized in some detail, showing quite mild phenotypic consequences [19,39]. By March 2020, there were 125 missense variants in the gnomAD v.2.1.1 database, whereas in the COSMIC database, there were 41 missense mutations compiled. Interestingly, only five mutations were found in both databases. Therefore, it is striking that so little is known about the functional implications of the genetic diversity of NQO1 in the human population.

In this work, we have investigated the effects of eight rare mutations on the stability and functional features of the NQO1 enzyme using biophysical, structural and bioinformatic approaches. Five mutations were found through high-throughput whole-genome/exome sequencing initiatives (gnomAD) and not particularly associated with cancer development and three are derived from cancer cell lines (COSMIC) (Table 1). These mutations affect residues located in the $\mathrm{N}$-terminal region of NQO1 and show different degrees of evolutionary conservation and expected impact on NQO1 stability and function. L7, T16 and Y20 are fully conserved among mammalian species (Figure 1) while V9 and A29 are highly conserved and G3 is not. Actually, the mutation A29T is found in some mammalian sequences while G3 is not even the preferred (consensus) residue. We may only consider the mutation V9I as conservative, since the rest of mutations will cause large changes in either backbone conformation/flexibility or side-chain size, polarity or charge. Structurally, residues affected by these mutations are located in the most N-terminal loop (L1, Gly3), a fully buried short $\beta$-sheet ( $\beta 1$, Leu7 and Val9), a solvent-exposed loop (L2, Thr16) and a long $\alpha$-helix ( $\alpha 1$, solvent-exposed Tyr20 and buried Ala29) (Figure 1A,B). Initial structure-based assessments for their impact on NQO1 stability support that V9I and T16M have neutral effects $\left(\Delta \Delta \mathrm{G}<-0.5 \mathrm{kcal} \cdot \mathrm{mol}^{-1}\right)$ and G3S, G3D, Y20N and A29T are destabilizing $\left(\Delta \Delta \mathrm{G} \sim-1-1.5 \mathrm{kcal} \cdot \mathrm{mol}^{-1}\right)$, while L7P and L7R are very destabiling $\left(\Delta \Delta \mathrm{G} \geq-2 \mathrm{kcal} \cdot \mathrm{mol}^{-1}\right)$ (note that these algorithms do not consider the effect of bound ligands in the structure). 

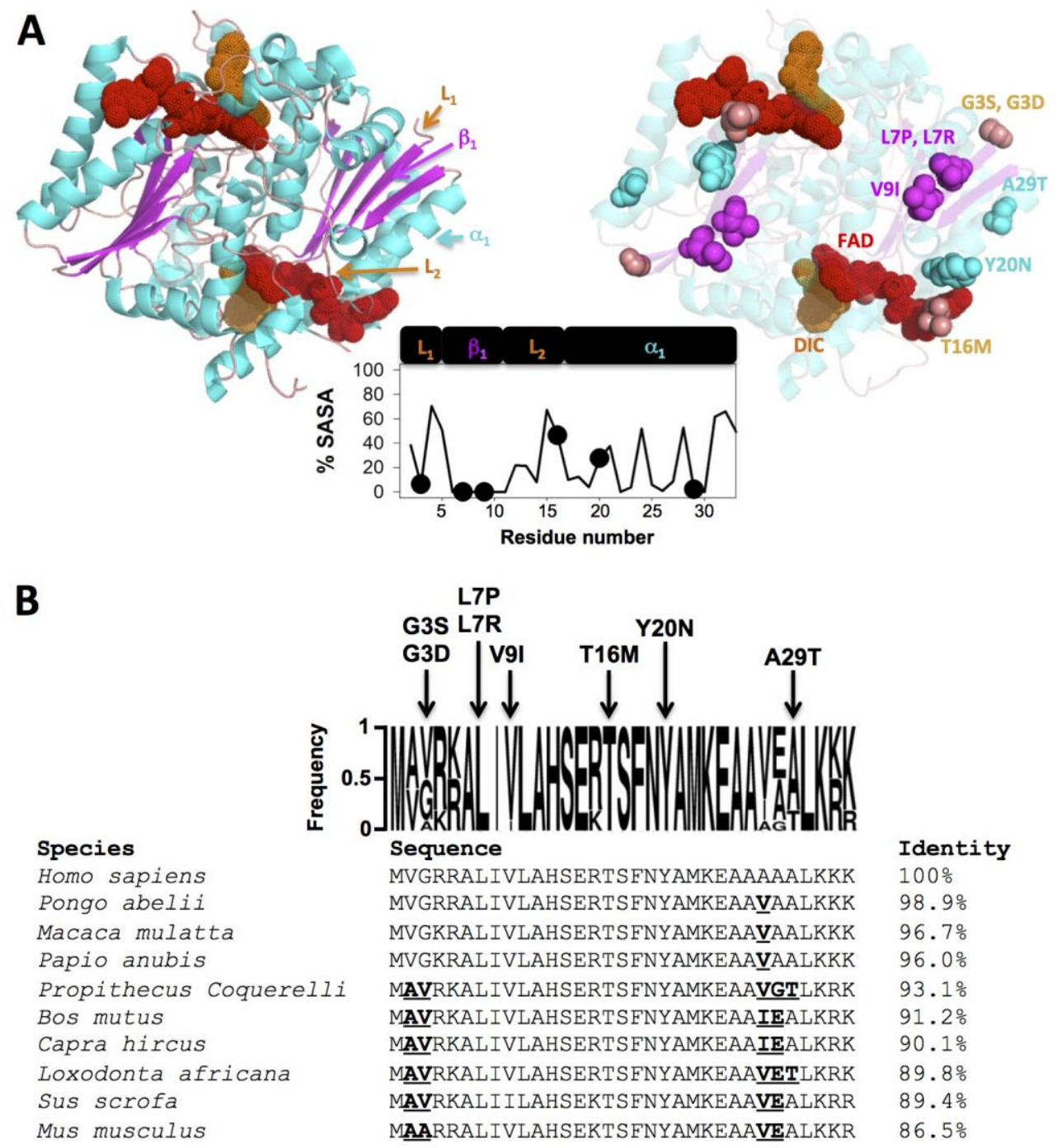

Figure 1. Structural location and sequence conservation of the NQO1 mutations studied in this work. (A) Structural representation on the dimeric structure of NQO1 (PDB code 2F1O; [72]). The left panel shows the secondary structure elements to which mutated residues belong $\left(\mathrm{L}_{1}\right.$, residues 1-4; $\beta_{1}$, residues $5-10 ; L_{2}$, residues $11-16$ and $\alpha_{1}$, residues $17-33$ ). The right panel shows the location of the mutated residues as well as the FAD and dicoumarol (Dic) molecules. Residues are colored according to the secondary structure. The plot in the middle shows the solvent accessibility (\% SASA) of this region (determined using GETAREA on the PDB code $2 \mathrm{~F} 1 \mathrm{O}$ as the average of the two monomers in the dimer). (B) Sequence alignment of 10 selected NQO1 mammalian proteins. Residues in bold-underlined indicate non-conservative mutations vs. the human sequence (note that K-to- $\mathrm{R}$ or V-to-I are considered as conservative). The frequency plot over the NQO1 alignment (generated using WebLogo, https://weblogo.berkeley.edu/) also shows the identity of the missense mutations investigated in this work. 
Table 1. NQO1 mutations experimentally characterized in this work.

\begin{tabular}{|c|c|c|c|c|c|c|c|}
\hline \multirow{2}{*}{$\begin{array}{l}\text { Trivial } \\
\text { Name }\end{array}$} & \multirow{2}{*}{$\begin{array}{l}\text { Amino Acid } \\
\text { Change }\end{array}$} & \multirow{2}{*}{$\begin{array}{l}\text { Nucleotide } \\
\text { Change }\end{array}$} & \multirow[b]{2}{*}{ COSMIC } & \multicolumn{3}{|c|}{ gnomAD V.2.1.1 } & \multirow{2}{*}{$\begin{array}{c}\Delta \Delta \mathrm{G} \\
\left(\mathrm{kcal} \cdot \mathrm{mol}^{-1}\right)^{1}\end{array}$} \\
\hline & & & & $\begin{array}{l}\text { All Samples } \\
\text { (AS) }\end{array}$ & $\begin{array}{l}\text { Non-Cancer } \\
\text { (NC) }\end{array}$ & $\begin{array}{c}\text { Ratio } \\
\text { (AS:NC) }\end{array}$ & \\
\hline G3S & p.G3S & c. $7 \mathrm{G}>\mathrm{A}$ & - & $1.2 \times 10^{-5}$ & $1.3 \times 10^{-5}$ & 0.9 & $-1.5 \pm 1.0$ \\
\hline G3D & p.G3D & c. $8 \mathrm{G}>\mathrm{A}$ & + & - & - & - & $-1.4 \pm 0.9$ \\
\hline L7P & p.L7P & c. $20 \mathrm{~T}>\mathrm{C}$ & + & - & - & - & $-4.3 \pm 1.2$ \\
\hline L7R & p.L7R & c. $20 \mathrm{~T}>\mathrm{G}$ & - & $7.8 \times 10^{-5}$ & $7.5 \times 10^{-5}$ & 1.0 & $-2.1 \pm 0.8$ \\
\hline V9I & p.V9I & c. $25 \mathrm{G}>\mathrm{A}$ & - & $3.9 \times 10^{-5}$ & $4.1 \times 10^{-5}$ & 0.9 & $-0.4 \pm 0.6$ \\
\hline $\mathrm{T} 16 \mathrm{M}$ & p.T16M & c. $47 \mathrm{C}>\mathrm{T}$ & - & $2.8 \times 10^{-5}$ & $2.6 \times 10^{-5}$ & 1.1 & $-0.1 \pm 0.2$ \\
\hline Y20N & p.Y20N & c. $58 \mathrm{~T}>\mathrm{A}$ & - & $2.1 \times 10^{-5}$ & $1.9 \times 10^{-5}$ & 1.1 & $-1.1 \pm 0.6$ \\
\hline $\mathrm{A} 29 \mathrm{~T}$ & p.A29T & c. $85 \mathrm{G}>\mathrm{A}$ & + & - & - & - & $-1.3 \pm 1.3$ \\
\hline
\end{tabular}

\section{Materials and Methods}

\subsection{Protein Expression and Purification}

Mutations were introduced by site-directed mutagenesis on wild-type (WT) NQO1 cDNA cloned into the pET-15b vector (pET-15b-NQO1) by GenScript (Leiden, The Netherlands). Codons were optimized for expression in Escherichia coli and mutagenesis was confirmed by sequencing the entire cDNA. pET-15b-NQO1 plasmids were used to transform BL21(DE3) cells for protein expression. Typically, $40 \mathrm{~mL}$ of Luria-Bertani (LB) medium containing $0.1 \mathrm{mg} \cdot \mathrm{mL}^{-1}$ ampicillin was inoculated with transformed cells and grown for $16 \mathrm{~h}$ at $37^{\circ} \mathrm{C}$. These cultures were diluted into $800 \mathrm{~mL}$ of LB containing $0.1 \mathrm{mg} \cdot \mathrm{mL}^{-1}$ ampicillin (Canvax Biotech), grown at $37{ }^{\circ} \mathrm{C}$ for $3 \mathrm{~h}$ to reach an optical density of about 1.0 and then these were transferred to $25^{\circ} \mathrm{C}$ and induced with $0.5 \mathrm{mM}$ isopropyl $\beta$-d-1-thiogalactopyranoside (IPTG, Canvax Biotech). After $6 \mathrm{~h}$, cells were harvested by centrifugation and frozen at $-80{ }^{\circ} \mathrm{C}$ for $16 \mathrm{~h}$. Cells were resuspended in binding buffer, $\mathrm{BB}(20 \mathrm{mM}$ Na-phosphate, $300 \mathrm{mM} \mathrm{NaCl}, 50 \mathrm{mM}$ imidazole, pH 7.4) plus $1 \mathrm{mM}$ phenylmethylsulfonyl fluoride (PMSF, Sigma-Aldrich) and sonicated in an ice bath. These total extracts were centrifuged (2000× $g$, $30 \mathrm{~min}, 4^{\circ} \mathrm{C}$ ) and the supernatants (soluble extracts) were loaded into immobilized-metal affinity chromatography (IMAC) columns (Ni-Sepharose, GE Healthcare), washed with 30-40 volumes of $\mathrm{BB}$ and eluted in this buffer containing $500 \mathrm{mM}$ imidazole. These eluates were immediately buffer exchanged using PD-10 columns to the storage buffer (50 mM HEPES-KOH pH 7.4) and concentrated to $1 \mathrm{~mL}$ (purified NQO1 proteins). After centrifugation for $20 \mathrm{~min}$ at $21,000 \times \mathrm{g}$ at $4{ }^{\circ} \mathrm{C}$, the amount of protein (in NQO1 subunit) and the FAD content were determined from their UV-visible spectra using: $\varepsilon_{(280)}=47,900 \mathrm{M}^{-1} \cdot \mathrm{cm}^{-1}$ for NQO1 and $\varepsilon_{(450)}=11,300 \mathrm{M}^{-1} \cdot \mathrm{cm}^{-1}$ for FAD [43]. Spectra were collected in a Cary 50 spectrophotometer (Agilent) using $0.3 \mathrm{~cm}$ path-length quartz cuvettes. The amount of FAD per NQO1 monomer was determined from the ratio between the absorbance at $450 \mathrm{~nm}$ from normalized spectra (in $\mathrm{M}^{-1} \cdot \mathrm{cm}^{-1}$ ) and the extinction coefficient of FAD (thus assuming that the FAD spectral properties bound to NQO1 resemble those of the free flavin). The lack of significant light scattering (due protein aggregation) was routinely checked. Each NQO1 variant was expressed and analyzed at least three times. The purity of purified NQO1 proteins was checked by SDS-PAGE in 12\% acrylamide gels.

The amount of NQO1 in total and soluble extracts was determined by Western-blot. Samples were resolved in 12\% acrylamide SDS-PAGE gels and electrotransfered to polyvinylidene difluoride (PVDF) membranes (GE Healthcare) using standard procedures. Immunoblotting was carried out using a primary antibody against NQO1 (F8, sc-393736, at 1:500 dilution) and, as secondary antibody, an anti-mouse IgGK BP-HRP antibody (sc-516102, at 1:2000 dilution) (both antibodies were purchased from Santa Cruz Biotechnology). Samples were visualized using luminol-based enhanced 
chemiluminiscence (from BioRad Laboratories). Densitometric analyses were performed using Image Lab (BioRad Laboratories) and ImageJ (http://rsb.info.nih.gov/ij/).

To obtain apo-proteins, FAD was removed by treatment with $2 \mathrm{M} \mathrm{KBr}$ and $2 \mathrm{M}$ urea, $1 \mathrm{mM}$ $\beta$-mercapto-ethanol in BB and PMSF $1 \mathrm{mM}$, as previously described [43,44]. Apo-proteins were buffer exchanged to $50 \mathrm{mM}$ HEPES-KOH $\mathrm{pH}$ 7.4. All purified proteins were stored at $-80^{\circ} \mathrm{C}$ upon flash-freezing in liquid nitrogen.

\subsection{In Vitro Characterization of Purified Proteins}

For thermal denaturation experiments, purified NQO1 proteins were prepared at a $2 \mu \mathrm{M}$ final concentration in monomer in the presence of a 10-fold excess of FAD in $50 \mathrm{mM}$ HEPES-KOH, $\mathrm{pH}$ 7.4. Triplicate samples were loaded into $3 \times 3 \mathrm{~mm}$ path-length quartz cuvettes. Thermal denaturation was carried out in a Cary Eclipse (Varian) spectrofluorimeter equipped with a Peltier element. The instrument was calibrated to correct the operational temperature provided by the instrument and the real one using a thermocouple. Samples were equilibrated at $20^{\circ} \mathrm{C}$ for $3 \mathrm{~min}$ and temperature was increased up to $70^{\circ} \mathrm{C}$ at a $2{ }^{\circ} \mathrm{C} \cdot \mathrm{min}^{-1}$ scan rate. Fluorescence emission was recorded at $350 \mathrm{~nm}$ (slit $10 \mathrm{~nm}$ ) upon excitation at $280 \mathrm{~nm}$ (slit $5 \mathrm{~nm}$ ). Experimental curves were normalized using pre- and post-transition linear baselines to yield the apparent half-denaturation temperatures $\left(T_{m}\right)$. For each variant, triplicate measurements were carried out and analyzed using two different protein batches. Thus, data are presented as mean \pm s.d. from six experiments for each variant.

For partial proteolysis experiments, thermolysin from Geobacillus stearothermophilus (Sigma-Aldrich, Madrid, Spain) was prepared in $50 \mathrm{mM}$ HEPES-KOH, pH 7.4, with $100 \mathrm{mM} \mathrm{CaCl}_{2}$. The concentration of thermolysin stock solutions was determined spectrophotometrically using $\varepsilon_{280}=66,086 \mathrm{M}^{-1} \cdot \mathrm{cm}^{-1}$ and small aliquots were stored at $\sim 20 \mu \mathrm{M}$ until used. For experiments, purified NQO1 proteins were prepared at $\sim 10 \mu \mathrm{M}$ NQO1 with $100 \mu \mathrm{M}$ FAD in $50 \mathrm{mM}$ HEPES-KOH, pH 7.4 (all concentrations were final concentrations), incubated for $5 \mathrm{~min}$ at $25^{\circ} \mathrm{C}$ and reactions were initiated by addition of thermolysin (to $0.2-1.2 \mu \mathrm{M}$, final concentration of $10 \mathrm{mM} \mathrm{CaCl}_{2}$ ). Aliquots were withdrawn at different times, mixed with $25 \mathrm{mM}$ ethylenediaminetetraacetic acid (EDTA), pH 8, and denatured at $95^{\circ} \mathrm{C}$ with Laemmli buffer. Controls without thermolysin were prepared similarly and considered as samples for time zero. Samples were resolved in SDS-PAGE gels (12\% acrylamide) and analyzed by densitometry using ImageJ. The decay of the full-length protein was fitted using a single exponential function to provide the first-order kinetic constant $k_{\mathrm{obs}}$, whereas the second-order rate constant $k_{\text {prot }}$ was determined by calculating the slope of the linear fit of $k_{\mathrm{obs}}$ vs. thermolysin concentration. Changes in local stability of the primary cleavage site upon mutation $\Delta \Delta \mathrm{G}_{\text {prot(WT-mut) }}$ were determined by the following equation [73]:

$$
\Delta \Delta G_{\text {prot }(\text { WT-mut })}=R \cdot T \cdot \ln \frac{k_{\text {prot }(m u t)}}{k_{\text {prot }(W T)}}
$$

where $R$ is the ideal gas constant, $T$ is the absolute temperature and $k_{\text {prot(mut) }}$ and $k_{\text {prot(WT) are }}$ the second-order rate constants for the mutant and the wild-type protein, respectively. Errors in $\Delta \Delta \mathrm{G}_{\text {prot(WT-mut) }}$ are those determined by linear propagation of the fitting errors for the $k_{\text {prot }}$ values.

Fluorescence titrations were carried out at $25^{\circ} \mathrm{C}$ using $1 \times 0.3 \mathrm{~cm}$ path-length cuvettes in a Cary Eclipse spectrofluorimeter (Agilent Technologies, Madrid, Spain). Experiments were carried out in 20 mM K-phosphate, pH 7.4, to decrease the binding affinity of NQO1 for FAD and to allow more accurate characterization of the affinity in tightly binding variants [42]. Then, $20 \mu \mathrm{L}$ of a $12.5 \mu \mathrm{M}$ NQO1 stock solution (in subunit) was mixed with $0-500 \mu \mathrm{L}$ of FAD $10 \mu \mathrm{M}$ and the corresponding volume of buffer was added to yield a $1 \mathrm{~mL}$ final volume. Samples were incubated at $25^{\circ} \mathrm{C}$ in the dark for at least $10 \mathrm{~min}$ before measurements. Fluorescence spectra were acquired in the $340-360 \mathrm{~nm}$ range upon excitation at $280 \mathrm{~nm}$ (slits $5 \mathrm{~nm}$ ), and spectra were averaged over 10 scans registered at a scan rate of $200 \mathrm{~nm} \cdot \mathrm{min}^{-1}$. Blanks were also measured similarly (containing only buffer) and subtracted. 
The intensity of the fluorescence at $350 \mathrm{~nm}$ (I) vs. total concentration of FAD ([FAD]) was used to determine the apparent dissociation constant $K_{\mathrm{d}(\mathrm{FAD})}$ using the following equation:

$$
I=I_{\text {apo }}+\left(I_{\text {holo }}-I_{\text {apo }}\right) \cdot\left(\frac{\left[N Q O 1_{\text {apo }}\right]+[F A D]+K_{d(F A D)}-\sqrt{\left([N Q O 1]+[F A D]+K_{d(F A D)}\right)^{2}-4 \cdot[N Q O 1] \cdot[F A D]}}{2 \cdot[N Q O 1]}\right)
$$

where $\mathrm{I}_{\text {holo }}$ and $\mathrm{I}_{\text {apo }}$ are the fluorescence intensity of $\mathrm{NQO}_{\text {holo }}$ and $\mathrm{NQO}_{\text {apo }}$, respectively, and [NQO1] is the total protein concentration $(250 \mathrm{nM})$. Data from at least two independent titrations using different preparations of apo-proteins for each NQO1 variant were used in the fitting. The apparent change in binding free energy, $\Delta \mathrm{G}_{(\mathrm{FAD})}$, was determined as follows: $\Delta \mathrm{G}_{(\mathrm{FAD})}=\mathrm{R} \cdot \mathrm{T} \cdot \ln K_{\mathrm{d}(\mathrm{FAD})}$. The error associated with $\Delta \mathrm{G}_{(\mathrm{FAD})}$ was determined by linear propagation from the errors of $K_{\mathrm{d}(\mathrm{FAD})}$.

Titrations of $\mathrm{NQO}_{\text {holo }}$ proteins with dicoumarol (Dic) were carried out by isothermal titration calorimetry (ITC) in an ITC $_{200}$ microcalorimeter (Malvern, Iesmat, Alcobendas, Spain). Purified NQO1 samples were prepared in $50 \mathrm{mM}$ HEPES-KOH, pH 7.4, in the presence of $100 \mu \mathrm{M}$ FAD and loaded into the calorimetric cell. Dic solutions were prepared from $10 \mathrm{mM}$ stocks (in $100 \mathrm{mM} \mathrm{NaOH}$ ) and diluted into $50 \mathrm{mM}$ HEPES-KOH pH 7.4 to a final concentration in Dic of $120 \mu \mathrm{M}$ (and $100 \mu \mathrm{M}$ FAD) and loaded in the titrating syringe. Experiments were typically carried out by performing an initial injection of $0.5 \mu \mathrm{L}$ followed by 20-22 injections of $1.75 \mu \mathrm{L}$, spaced by $100 \mathrm{~s}$. The instrument operated in the high-feedback mode. Data analysis was carried out upon manual integration of the experimental enthalpograms and fittings were done using a single type of independent binding sites model with the software provided by the manufacturer. Dilution heats were included as a fitting parameter. This analysis yields the number of bindings per NQO1 monomer $(\mathrm{N})$ as well as the association binding constant $\left(K_{\mathrm{a}}\right.$, being $\left.K_{\mathrm{a}}=1 / K_{\mathrm{d}}\right)$, enthalpy $(\Delta \mathrm{H})$ and entropy $(\Delta \mathrm{S})$. Experiments were performed at least three times at $25{ }^{\circ} \mathrm{C}$ and using two different protein preparations of each variant. Data at this temperature are presented as mean \pm s.d. Experiments at $10,15,17.5$ and $20^{\circ} \mathrm{C}$ were performed only once and reported errors were those from fittings. The apparent change in heat capacity $\left(\Delta C_{p}\right)$ was determined from linear dependence of $\Delta \mathrm{H}$ on temperature.

Structure-energetic relationships were combined with Dic binding calorimetric data to determine the magnitude of the conformational change associated with binding $\left(\mathrm{N}_{\text {conf }}\right.$, in number of residues) using previously described procedures [39]. Briefly, two experimental apparent thermodynamic binding variables, $\Delta \mathrm{H}$ and $\Delta \mathrm{C}_{\mathrm{p}}$, were assumed to be the sum of two separate contributions: one arising from the energetics of rigid-body interactions between $N Q O 1_{\text {holo }}$ and Dic (intrinsic binding) and the other arising from the conformational change associated to binding. The former can be estimated from changes in solvent accesible surface upon Dic binding as determined from the crystal structure of the $\mathrm{NQO}_{\text {holo }}$ complex with Dic (NQO1 $1_{\text {dic }}$; PDB code $2 \mathrm{~F} 1 \mathrm{O}$, [72]), yielding values of $-5.8 \mathrm{kcal} \cdot \mathrm{mol}^{-1}$ and $-0.14 \mathrm{kcal} \cdot \mathrm{mol}^{-1} \cdot \mathrm{K}^{-1}$ for intrinsic $\Delta \mathrm{H}\left(\right.$ at $25^{\circ} \mathrm{C}$ ) and $\Delta \mathrm{C}_{\mathrm{p}}$, respectively. Thus, the contribution from the conformational change $\left(\Delta \mathrm{H}_{\text {conf }}\right.$ and $\left.\Delta \mathrm{C}_{\mathrm{p}, \mathrm{conf}}\right)$ can be obtained from the difference between the experimental variables and those calculated for intrinsic binding. This contribution can be parametrized in terms of the number of residues folded upon binding (i.e., those involved in the conformational change) from well-known structure-energetics relationships for protein folding as follows [39,74]:

$$
\begin{aligned}
\Delta \mathrm{H}_{\mathrm{conf}} & =0.215 \cdot \mathrm{N}_{\mathrm{conf}} \\
\Delta \mathrm{C}_{\mathrm{p}, \mathrm{conf}} & =0.0138 \cdot \mathrm{N}_{\mathrm{conf}}
\end{aligned}
$$

where $\Delta \mathrm{H}_{\text {conf }}$ is given in $\mathrm{kcal} \cdot \mathrm{mol}^{-1}$ and $\Delta \mathrm{C}_{\mathrm{p}, \mathrm{conf}}$ in $\mathrm{kcal} \cdot \mathrm{mol}^{-1} \cdot \mathrm{K}^{-1}$. This approach provides two ways of determining $\mathrm{N}_{\text {conf }}$ from experimental and structural variables.

\subsection{In Silico Mutagenesis and Structural Analysis}

In silico mutagenesis of the dimeric NQO1 protein was performed using Rosetta [75]. The atomic coordinate files for the $\mathrm{NQO}_{\text {holo }}$ state (PDB code $1 \mathrm{D} 4 \mathrm{~A}$ ) and $\mathrm{NQO}_{\text {dic }}$ state (PDB code $2 \mathrm{~F} 1 \mathrm{O}$ ) of the 
WT protein were processed to remove non-canonical amino acids and water molecules. Subsequently, each PDB file was refined with fast relax constrained to native coordinates using Cartesian-space refinement as described elsewhere [76] and the Rosetta energy function REF15. After relaxation and refinement, the structural model with the lowest energy was used as the starting structure for mutagenesis. For in silico mutagenesis, the cartesian version of Rosetta's ddG protocol [77] was followed and five structures were generated for each variant (WT and eight mutants), both in the $\mathrm{NQO}_{\text {holo }}$ and $\mathrm{NQO}_{\text {dic }}$ states, following the procedures described in [76]. Finally, the energies (expressed in $\mathrm{kcal} \cdot \mathrm{mol}^{-1}$ ) of every structure were calculated and averaged for each variant in a given ligation state. $\Delta \Delta G$ values were calculated by subtracting the average energy of the corresponding mutant ensemble to the average energy of the WT ensemble. As cut-off, $\Delta \Delta \mathrm{G}$ values $>0.5 \mathrm{kcal} \cdot \mathrm{mol}^{-1}$ were considered as stabilizing, $\Delta \Delta \mathrm{G}$ values $<-0.5 \mathrm{kcal} \cdot \mathrm{mol}^{-1}$ were considered as destabilizing and $\Delta \Delta \mathrm{G}$ values between -0.5 and $+0.5 \mathrm{kcal} \cdot \mathrm{mol}^{-1}$ were considered as neutral. Structural analysis and representations were generated with YASARA molecular modeling software [78].

\subsection{Structure- and Sequence-Based Analysis of Mutational Effects on Protein Stability and Potential Pathogenicity}

To determine changes in protein (thermodynamic stability), we used the following methods: (i) SDM2 (Site Directed Mutator 2, http://marid.bioc.cam.ac.uk/sdm2/prediction) [79] analyses were carried out using chains A and B from three high-resolution crystal structures (PDB codes 2F1O, 1D4A and 5FUQ, $[43,72,80])$. For each mutant, the value provided was the mean \pm s.d. from six analyses; (ii) PoPMuSiC (https://soft.dezyme.com/) [81] analyses were carried out using all chains from three high-resolution crystal structures (PDB codes 2F1O, 1D4A and 5FUQ, [43,72,80]). For each mutant, the value provided was the mean \pm s.d. from three analyses; (iii) DynaMut (http://biosig. unimelb.edu.au/dynamut/) [82] analyses were carried out in chains A and C (PDB codes 2F1O and 1D4A, [72,80]) or chains A and B (PDB code 5FUQ, [43]). For each mutant, the value provided was the mean \pm s.d. from six analyses; (iv) MAESTRO (https://pbwww.che.sbg.ac.at/) $[83,84]$ analyses were carried out in chains A and C (PDB codes 2F1O and 1D4A, [72,80]) or chains A and B (PDB code $5 F U Q,[43])$. For each mutant, the value provided was the mean \pm s.d. from six analyses; (v) CUPSAT (http://cupsat.tu-bs.de/) [85] analyses were carried in the Thermal denaturation method using chains A and B from three high-resolution crystal structures (PDB codes 2F1O, 1D4A and 5FUQ, [43,72,80]). For each mutant, the value provided was the mean \pm s.d. from six analyses.

For bioinformatic prediction of pathogenicity, we used: (i) PolyPhen-2 (http://genetics.bwh. harvard.edu/pph2/index.shtml) analyses, which yielded the potential effect of mutations as Benign, Possibly damaging or Probably damaging, using information on multiple sequence alignments and simple physical estimations of mutational effects [32]; (ii) SIFT (https://sift.bii.a-star.edu.sg/) analyses, which used multiple sequence alignment tools to estimate evolutionary tolerance of mutations (Tolerated vs. Not tolerated) [86]; (iii) PROVEAN (http://provean.jcvi.org) analyses, which used a sequence-based (multiple sequence alignment) approach to predict functional effects of mutations (Neutral vs. Deleterious effect) [87]; (iv) PON-P2 (http://structure.bmc.lu.se/PON-P2) analyses, which provided mutational effects as Pathogenic, Neutral or Unknown using evolutionary sequence conservation, properties of amino acids and physical and functional annotations of replacements sites [88]; (v) MutationTaster (http:// www.mutationtaster.org) analyses, which yielded mutational effects as Polymorphisms or Disease-causing using DNA sequence alterations and information from human genome variability, evolutionary conservation, splice-site changes, loss of protein features and changes that might affect the amount of mRNA [89]. For methods $\mathrm{i}-\mathrm{iv}$, the input was the NQO1 protein sequence, and for method $v$, the NQO1 cDNA sequence. 


\section{Results and Discussion}

3.1. Expression Analysis of NQO1 Variants Reveals Dramatic Effects of the Mutations L7R and L7P on Protein Stability and/or Solubility

To characterize the effects of rare NQO1 mutations on the stability and functional features of this protein, we expressed them in E. coli and purified the WT and mutant proteins. Two of the mutants (L7R and L7P) showed largely reduced expression levels as soluble protein and were not amenable for purification, supporting that these two mutations severely perturb NQO1 folding and prevent the formation of stably folded dimers (Figure 2A,B). Accordingly, structure-based analysis of protein destabilization supported that these two mutations should be the most deleterious (Table 1). Previous expression analyses have also shown similar behavior for largely disrupting mutations at the P187 site (a residue buried in the structure and close to the MMI), such as P187R, P187E and P187L [19,39]. The remaining six mutants were amenable for purification and showed comparable yields to those of WT NQO1 (Figure 2B,C).

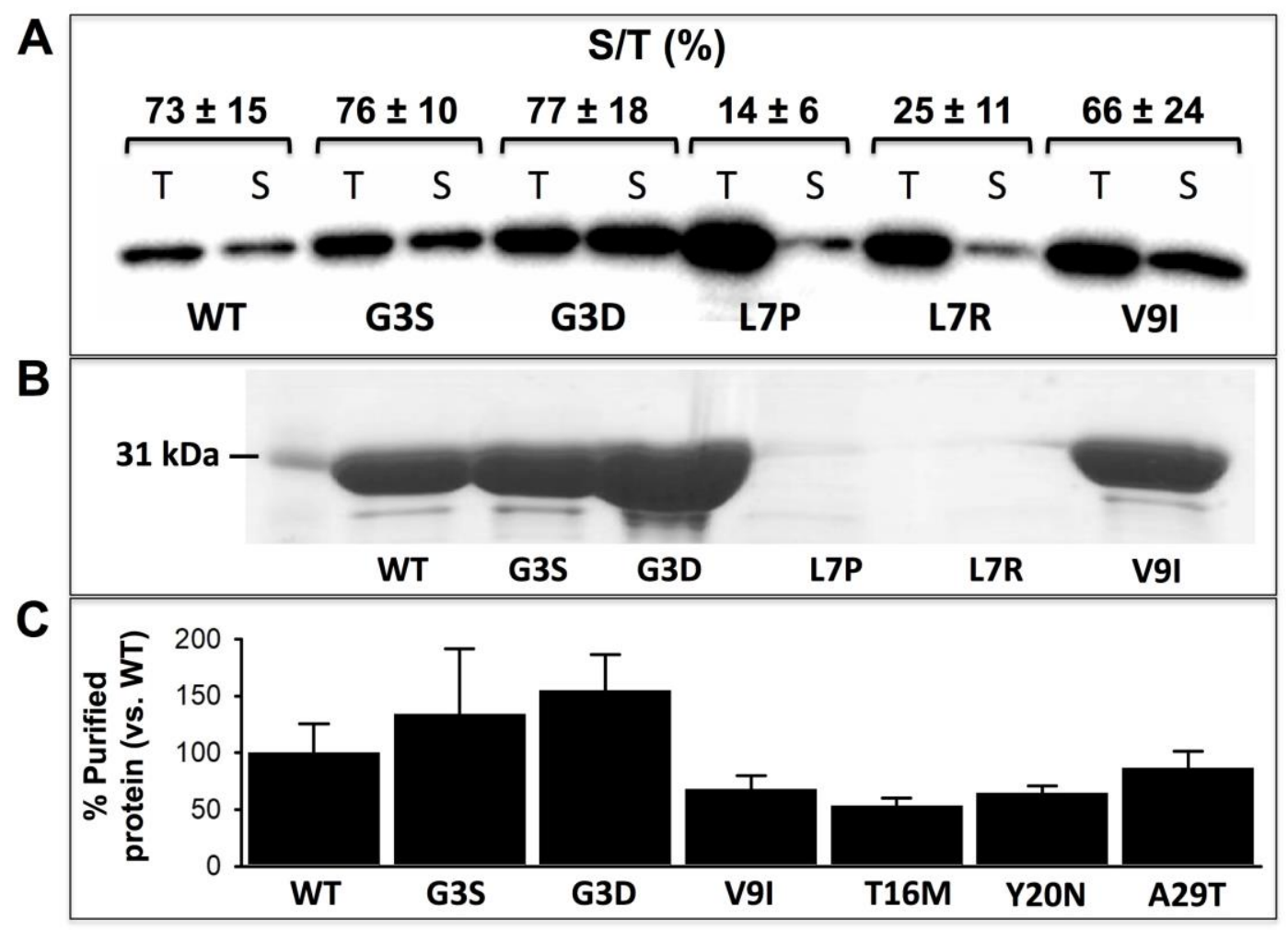

Figure 2. Expression and solubility of NQO1 variants in E. coli cells. (A) Western-blot analysis of NQO1 content upon expression in E. coli cells. Cells were sonicated to obtain total extracts (T), while soluble extracts (S) were obtained upon centrifugation at $21,000 \times \mathrm{g}$ for $20 \mathrm{~min}$ at $4{ }^{\circ} \mathrm{C}$. Samples were denatured with Laemmli buffer and submitted for Western-blot analysis using anti-NQO1 antibody, F-8 antibody (Santa Cruz Biotechnology). The Western-blot is representative from three different purifications. The fraction of the total protein found in the soluble extract upon densitometric analysis ( $\mathrm{S} / \mathrm{T}$, as \%) is indicated (as mean \pm s.d. from three independent experiments). (B) Purified NQO1 proteins from three different purifications were concentrated $\sim 10$-fold and samples analyzed by SDS-PAGE. Note that during purification and storage, the remaining soluble protein of L7P and L7R was negligible. (C) Yield in NQO1 protein variants after immobilized-metal affinity chromatography (IMAC) purification. Data were the mean \pm s.d. from 3-4 different purifications for each NQO1 variant. Wild-type (WT) levels were $1.45 \pm 0.38 \mathrm{mg} \cdot \mathrm{L}^{-1}$ of culture and used to normalize yields. 


\subsection{Thermal Stability Analyses Revealed Significant Perturbation of the MMI by Mutations T16M and Y20N}

For the six mutants amenable for purification, we analyzed and compared their stability with that of the WT protein in the presence of an excess of FAD ( $\mathrm{NQO1}_{\text {holo }}$ ) and using fluorescence-monitored thermal denaturation (Figure 3). To some extent, the thermal stability of NQO1 variants allows to capture the perturbation caused by the mutations on the MMI since the dimer dissociates prior to the rate-limiting step of the irreversible denaturation [15,39,41]. T16 and Y20 are within or in very close proximity to the MMI and mutations at these residues are potentially the most damaging for dimer stability (Figure 3A). Consequently, the mutations T16M and Y20N, which may introduce mild to moderate structural perturbations (Table 1), led to significant changes in thermal stability (i.e., their $\mathrm{T}_{\mathrm{m}}$ were lower than that of the WT protein by $4-5^{\circ} \mathrm{C}$ ) (Figure 3B,C). L7 and V9 are at the shortest distance of $8 \AA$ from the MMI (Figure 3A). Accordingly, the severely perturbing L7P and L7R mutation may significantly destabilize the MMI, thus explaining the dramatic effects of these mutations that prevent dimer formation or severely affect its stability (Figure 2). The mild effects of the mutation V9I likely reflect a small structural perturbation in some proximity to the MMI (reducing thermal stability by $1.5^{\circ} \mathrm{C}$; Figure $3 \mathrm{~B}, \mathrm{C}$ ). G3 and A29 are over $15 \AA$ away from the MMI (Figure 3A), and consequently, the effects of the mutations G3S, G3D and A29T in thermal stability were marginal (Figure 3B,C).
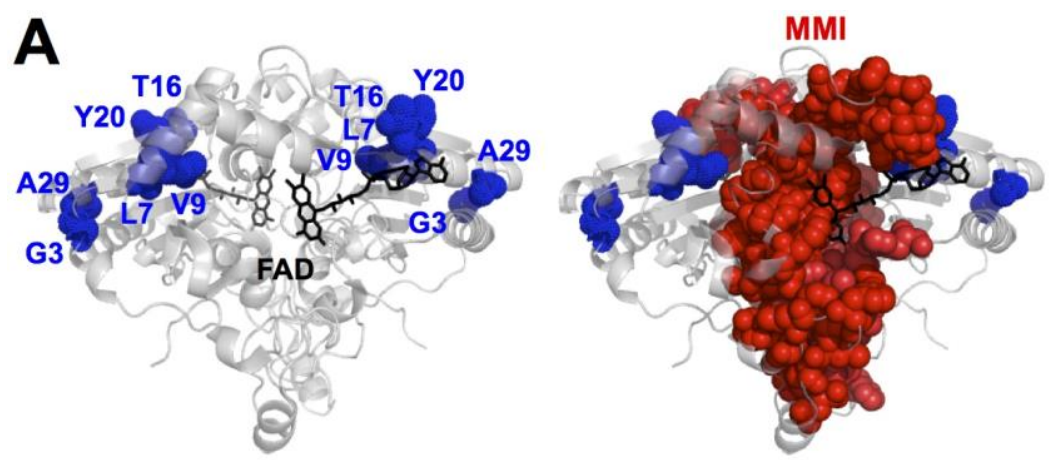

\begin{tabular}{|c|c|}
\hline Residue & $\begin{array}{c}\text { MMI } \\
(\mathbf{A})\end{array}$ \\
\hline Gly3 & 16.9 \\
\hline Leu7 & 8.0 \\
\hline Val9 & 7.2 \\
\hline Thr16 & 0 \\
\hline Tyr20 & 2.9 \\
\hline Ala29 & 15.0 \\
\hline
\end{tabular}
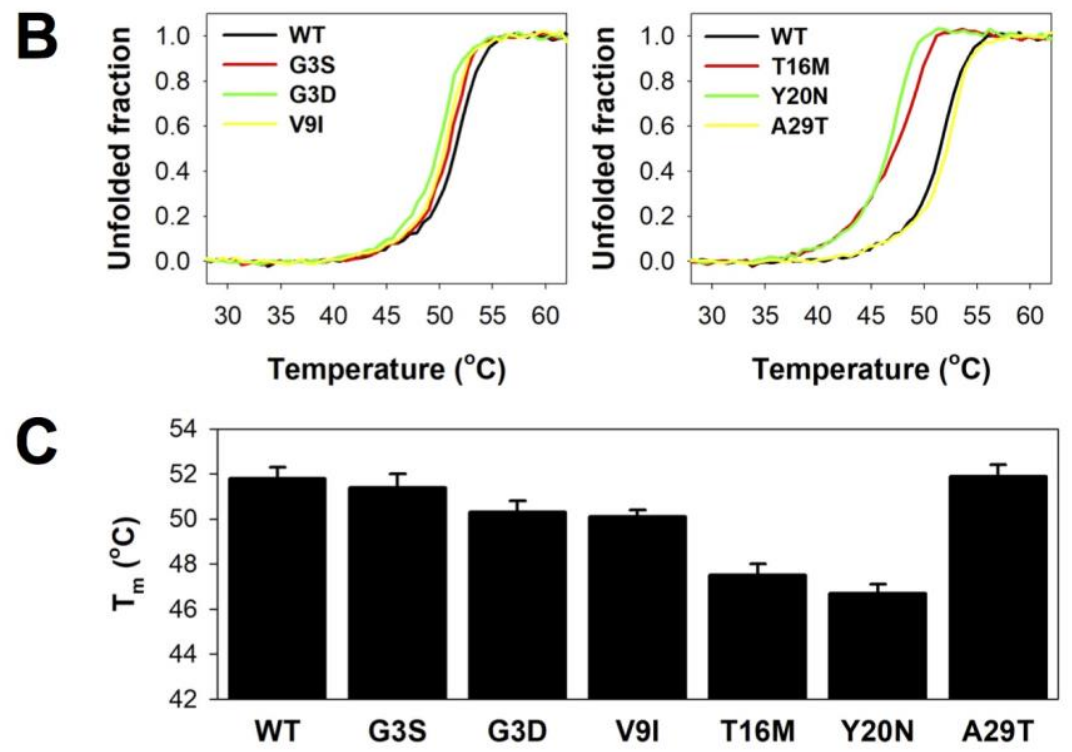

Figure 3. Thermal stability of NQO1 variants. (A) Structural location of the mutated residues, showing their proximity (as a minimal distance) to the monomer:monomer interface (MMI). The structure used for display has the PDB code 2F1O [72]. Residues belonging to the MMI were identified as described [90]. (B) Thermal denaturation profiles; (C) $\mathrm{T}_{\mathrm{m}}$ values (mean \pm s.d. from six replicas, using proteins from two purifications). 
It is important to note that the above-mentioned interpretation of mutational effects on NQO1 thermal stability simply implies that both the magnitude of the perturbation caused by the mutation and its distance-dependent propagation to the MMI are key features. Previous systematic mutational studies at the P187 and K240 residues of NQO1 generally supported this interpretation [39]. In the next sections, we will apply a similar approach to other features of NQO1, such as the mutational effects on local stability and binding of functional ligands.

\subsection{The Local Stability of the Thermolysin Cleavage Site (TCS) Is Reduced by the Distant Mutations T16M and $Y 20 N$}

Limited proteolysis of NQO1 is a sensitive method to probe the local stability at different regions of the protein $[41,50]$. In particular, proteolysis by thermolysin typically provides information on the local conformational stability of the region surrounding the primary cleavage site for this protease (i.e., the thermolysin cleavage site (TCS)) located in the NTD of NQO1 holo; it cleaves between S72-V73) [41]. For instance, the phosphomimetic mutation S82D locally destabilizes this region, accelerating 30-fold proteolysis by thermolysin of $\mathrm{NQO}_{\text {holo }}$ (i.e., it causes $2.0 \mathrm{kcal} \cdot \mathrm{mol}^{-1}$ of local destabilization in terms of $\Delta \mathrm{G}_{\text {prot }}$ as the mutational effect on the unfolding free energy between the native and cleavable states) [91]. It must be noted that all mutations studied in this work are located at least 15-20 $\mathrm{f}$ from the TCS (Figure 4A).
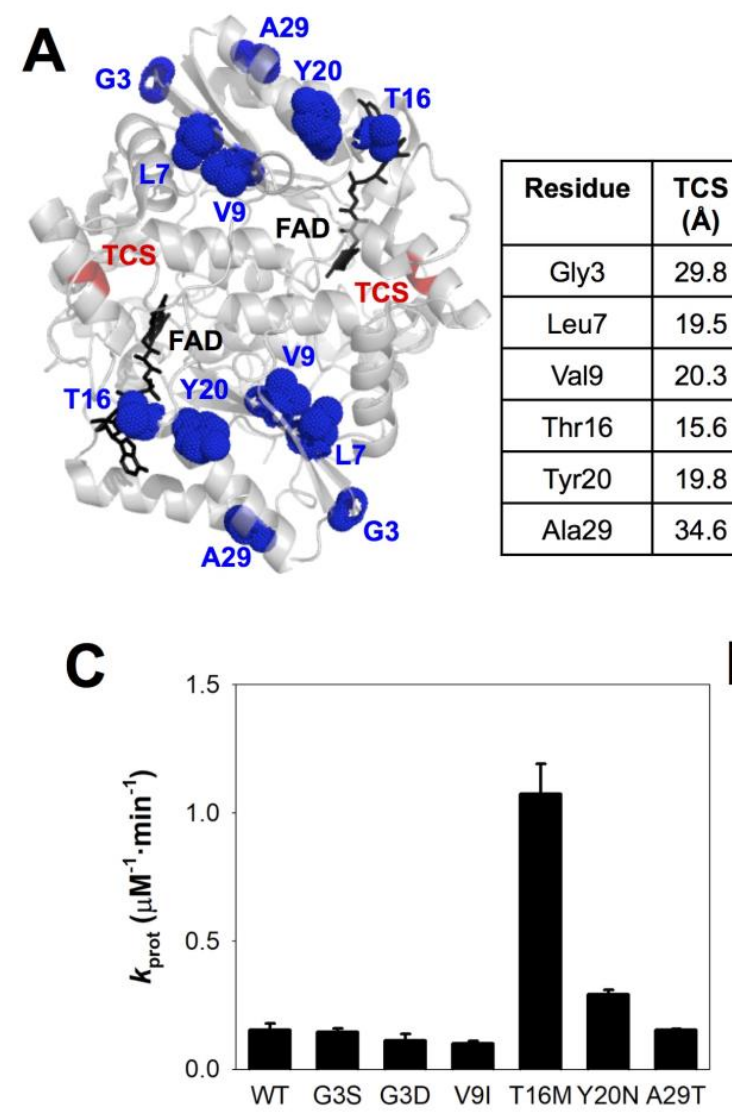

B

\begin{tabular}{|c|c|}
\hline Residue & $\begin{array}{c}\text { TCS } \\
(\mathbf{A})\end{array}$ \\
\hline Gly3 & 29.8 \\
\hline Leu7 & 19.5 \\
\hline Val9 & 20.3 \\
\hline Thr16 & 15.6 \\
\hline Tyr20 & 19.8 \\
\hline Ala29 & 34.6 \\
\hline
\end{tabular}

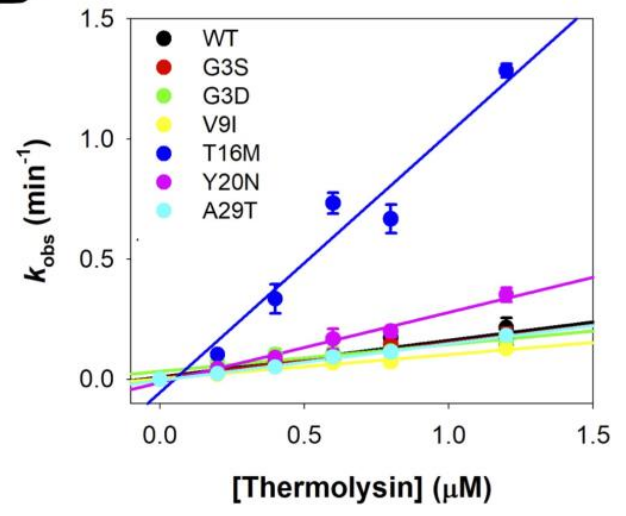

D

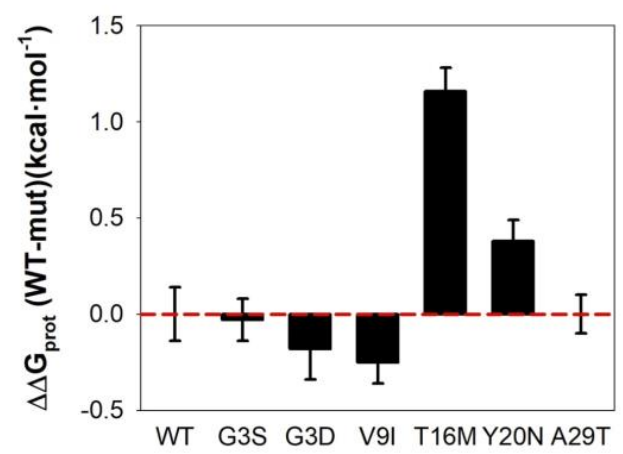

Figure 4. Local stability of the N-terminal domain (NTD) probed by proteolysis with thermolysin. (A) Location of mutated residues regarding the thermolysin cleavage site (TCS). The minimal distance between the mutated residue and the TCS backbone (Ser72-Val73) is also indicated (using the structure with PDB code $2 \mathrm{~F} 1 \mathrm{O})$. (B) Linear dependence of apparent rate constants $\left(k_{\mathrm{obs}}\right)$ on protease concentration. The slopes provide the values of the second-order rate constants $k_{\text {prot }}$ (C,D) Values of $k_{\text {prot }}$ for the NQO1 variants (C) and the effect of mutations on the local stability (D) as $\Delta \Delta G_{\text {prot }}$. Note that a positive value of $\Delta \Delta \mathrm{G}_{\text {prot }}$ indicates a destabilizing effect. 
Proteolytic patterns of all mutant $\mathrm{NQO}_{\text {holo }}$ proteins studied resembled that of WT NQO1 (Figure S1), supporting initial cleavage between S72-V73. Furthermore, proteolysis rate constants $\left(k_{\mathrm{obs}}\right)$ for all NQO1 variants showed a linear dependence on protease concentrations (Figure 4B and Figure S2), thus implying that changes on the second-order rate constant $k_{\text {prot }}$ are related to effects on the thermodynamic stability between the native and cleavable state in the TCS (Figure 4C,D) [41]. We found that only the mutations $\mathrm{T} 16 \mathrm{M}$ and $\mathrm{Y} 20 \mathrm{~N}$ caused local destabilization beyond the experimental error (by $\sim 1.1$ and $0.4 \mathrm{kcal} \cdot \mathrm{mol}^{-1}$, respectively; Figure $4 \mathrm{D}$ and Table S2). Thus, the structural perturbation introduced by these two mutations is sensed by the TCS, located $15-20 \AA$ away (Figure $4 \mathrm{~A}$ ).

\subsection{The Mutations T16M, Y20N and A29T Perturb FAD Binding}

The binding of FAD to NQO1 is very sensitive to structural perturbations caused by mutations in the $\mathrm{NQO}_{\text {apo }}$ and $\mathrm{NQO}_{\text {holo }}$ states [39-45,91]. Actually, when a single mutation reduces significantly FAD binding affinity (by at least 5 -fold, i.e., about $1 \mathrm{kcal} \cdot \mathrm{mol}^{-1}$ in binding free energy), this often decreases the FAD content of the protein upon purification from $E$. coli cell cultures $[39,40,43,44,91]$. Since bound FAD is mandatory for NQO1 catalytic function, these effects would affect the specific activity of the enzyme. Structurally, residues T16 and Y20 are close to the FAD molecule ( $\leq 5 \AA)$, whereas V9 and A29 are at 12-13 $\mathrm{A}$ and G3 is more than $25 \AA$ away (Figure 5A).

The FAD content in purified NQO1 proteins was determined using near-UV/visible absorption spectroscopy. All the mutants showed high levels of FAD bound, with fractions in the range 0.8-0.9 (similar to those of the WT protein), with the only exception of T16M that showed reduced content (0.5 mol FAD/NQO1 monomer, Figure 5B,C). To evaluate quantitatively mutational effects on FAD binding affinity, we carried out titrations of apo-proteins with FAD monitored by fluorescence spectroscopy (Figure 5D,E, Figure S3 and Table S3). As expected from its lower content in FAD as-purified, the mutant $\mathrm{T} 16 \mathrm{M}$ showed a 12 -fold lower affinity that corresponds to a decrease in binding free energy of about $1.5 \mathrm{kcal} \cdot \mathrm{mol}^{-1}$. In addition, the mutants $\mathrm{Y} 20 \mathrm{~N}$ and A29T showed decreased binding affinity (3- and 6-fold lower than that of WT NQO1, respectively, that correspond to changes in binding free energy of 0.7 and $1.0 \mathrm{kcal} \cdot \mathrm{mol}^{-1}$, respectively) (Figure 5C-E and Table S3). It is interesting to note the effect of the mutation $\mathrm{A} 29 \mathrm{~T}$, which involves a residue located at more than $13 \AA$ away from the FAD binding site.

\subsection{None of the Mutations Affect Dic Binding Affinity or Energetics}

Dic strongly inhibits NQO1 with a $K_{\mathrm{d}}$ of $10-50 \mathrm{nM}$, acting as a competitive inhibitor of the $\mathrm{NADP}(\mathrm{H})$ coenzyme and the substrate $[43,48,63,72,92]$. Investigating the affinity and energetics of Dic binding to NQO1 mutants may provide insight into mutational effects on several protein traits: (i) Dic (and analogues thereof) inhibits the growth of cancer cell lines, presumably by preventing the high antioxidant activity provided by cancer-associated overexpression of NQO1 $[63,93,94]$. Consequently, alterations of Dic binding due to missense mutations may yield decreased cellular sensitivity towards this potential pharmacological treatment for cancer; (ii) Missense mutations can decrease the binding affinity for Dic by altering the conformation and energetics of the native state, shifting the conformational equilibrium towards non-competent states for binding. An excellent example of this behavior is observed in the common polymorphic variant P187S that binds Dic with about a 10-fold lower affinity than the WT protein and shows clearly different binding energetics associated with the folding of the CTD upon Dic binding [39,44,92]; (iii) Extensive mutational studies at the P187 and K240 sites have shown that long-range communication (over $15 \AA$ from the binding site) of mutational effects acting on the conformation and stability of the CTD can be accurately characterized by a detailed analysis of Dic binding energetics $[19,39]$. As we show in Figure 6A, all the mutations investigated in this work are structurally located far from the Dic molecule (by at least $15 \AA$ ). 
A
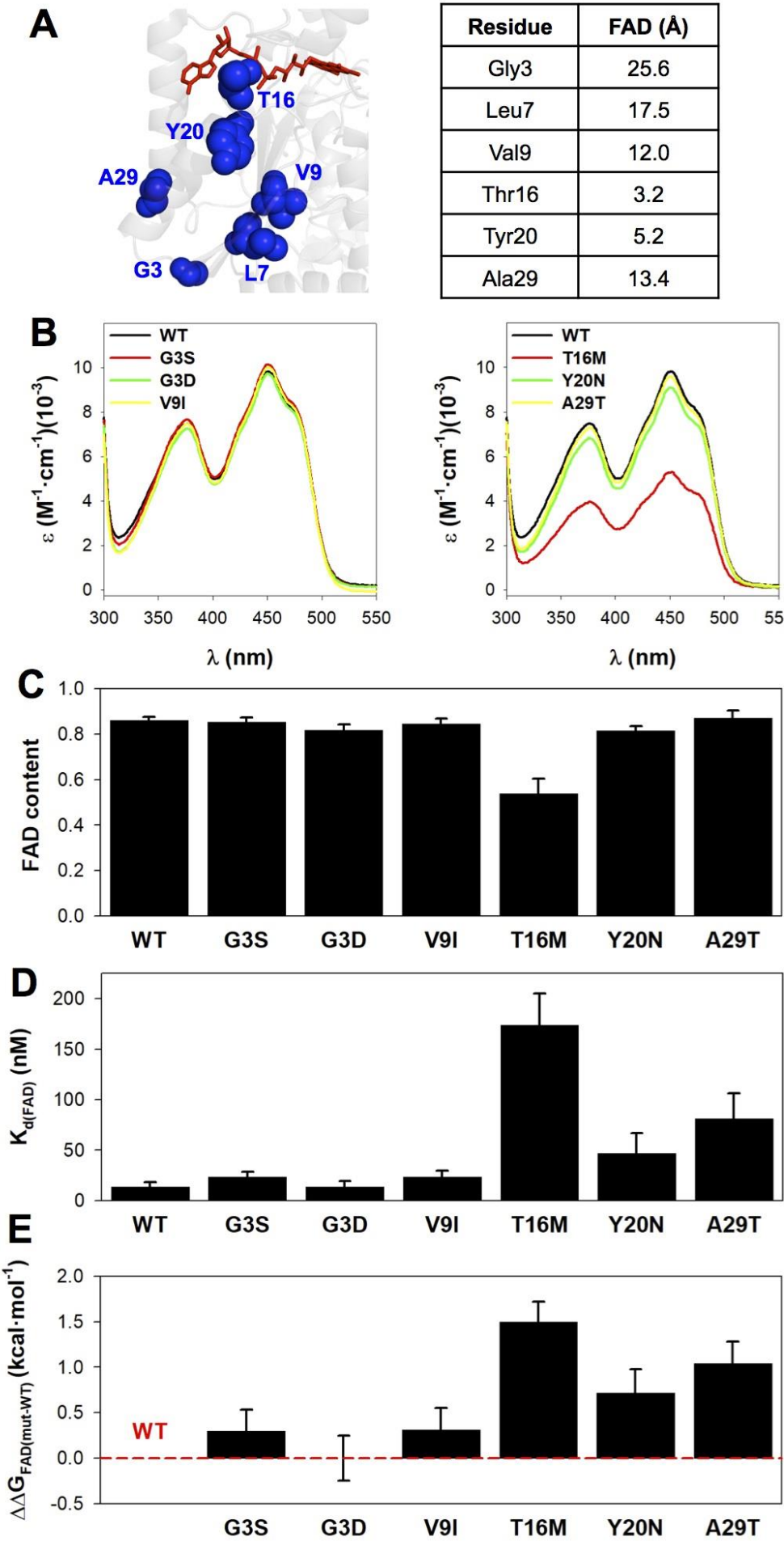

Figure 5. FAD content and binding affinity in purified NQO1 variants. (A) Location of mutated residues and minimal distances to the FAD molecule (using the structure with PDB code 2F1O). (B) Near-UV/visible absorption spectra of NQO1 variants. (C) FAD content (per NQO1 monomer) derived from absorption spectra (considering a $\varepsilon_{450}=11,300 \mathrm{M}^{-1} \cdot \mathrm{cm}^{-1}$ ). (D) FAD-binding affinity of $\mathrm{NQO}_{\text {apo }}$ proteins determined by fluorescence titrations. (E) Difference in binding free energies $\left(\Delta \Delta \mathrm{G}_{\mathrm{FAD}}\right)$ between a given mutant and the WT protein. Errors in $\Delta \Delta \mathrm{G}_{\mathrm{FAD}}$ are those determined from linear propagation. Data in B-C are mean \pm s.d. from at least three different purifications, and in D-E, from two different titrations with two different preparations of apo-proteins. 
A

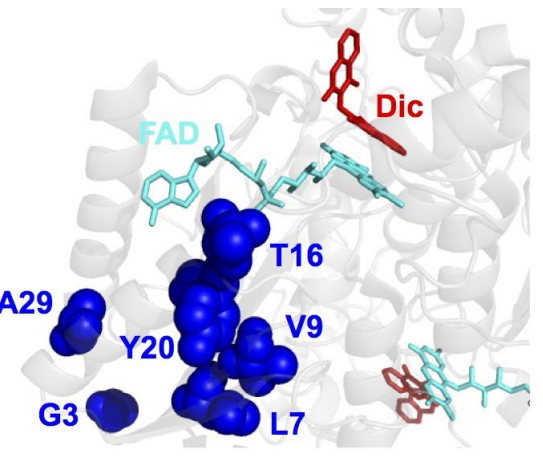

\begin{tabular}{|c|c|}
\hline Residue & Dic $(\boldsymbol{A})$ \\
\hline Gly3 & 26.2 \\
\hline Leu7 & 18.1 \\
\hline Val9 & 17.5 \\
\hline Thr16 & 16.3 \\
\hline Tyr20 & 16.8 \\
\hline Ala29 & 28.0 \\
\hline
\end{tabular}
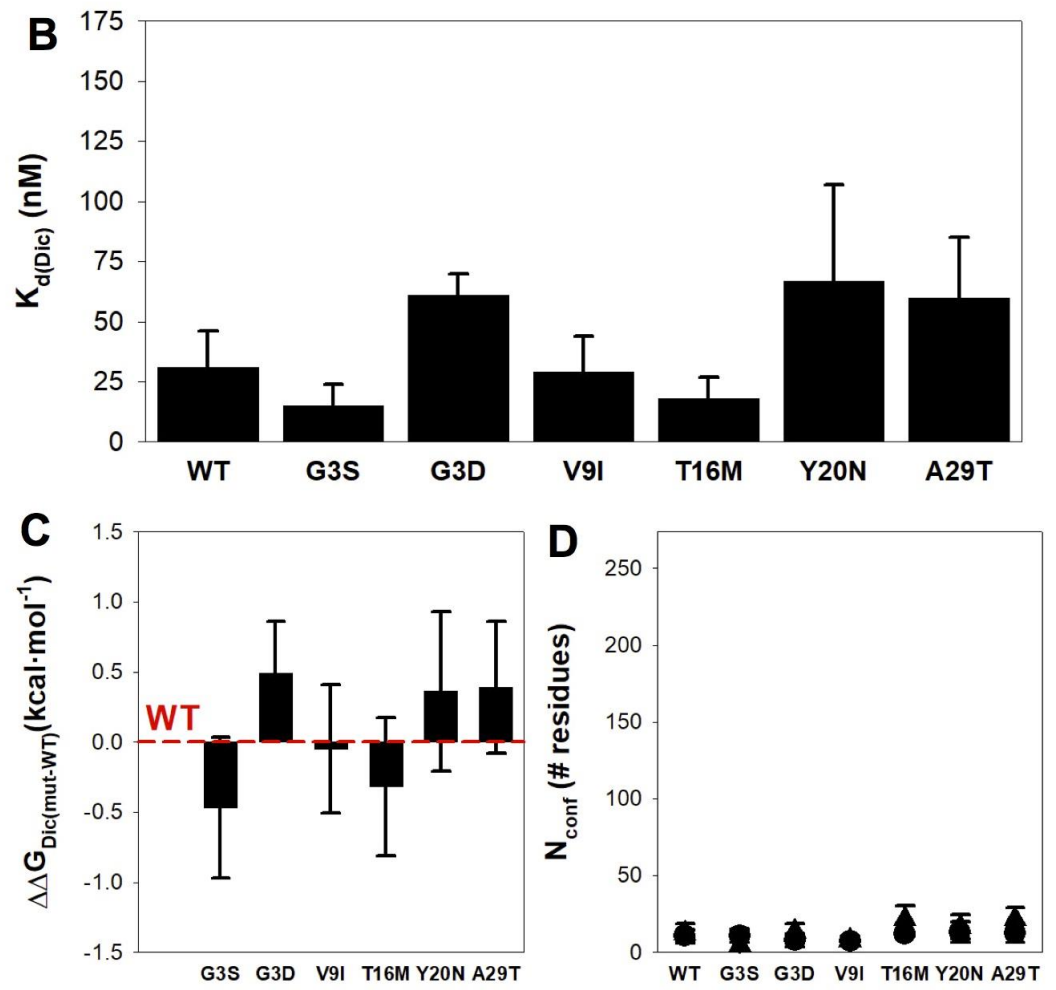

Figure 6. Affinity and structure-energetics analysis for Dic binding to NQO1 variants. (A) Location of mutated residues and minimal distances to Dic (using PDB code 2F1O). (B) Dissociation binding constants at $25^{\circ} \mathrm{C}$. Data are mean \pm s.d. from at least three independent experiments for each variant. (C) Difference in binding free energy between a given mutant and the WT protein. Errors are those determined from linear propagation. (D) Magnitude of the conformational change (as a number of residues, $\mathrm{N}_{\text {conf }}$ ) determined from experimental binding enthalpies (circles) and changes in heat capacity (triangles).

We have analyzed Dic-binding affinity and energetics by ITC (Figure 6 and Figure S4). All the NQO1 mutants bound Dic with an affinity comparable to that of NQO1 WT and close to the technical limit of the technique. The largest differences correspond to a mild 2-fold change, which implies changes in binding free energy $\leq 0.5 \mathrm{kcal} \cdot \mathrm{mol}^{-1}$ (Figure 6B,C and Table S4). The apparent enthalpic and entropic contributions to binding were also very similar (within the experimental error, see Figure S5 and Table S4). In addition, two apparent thermodynamic binding parameters ( $\Delta \mathrm{H}$ and $\left.\Delta \mathrm{C}_{\mathrm{p}}\right)$ can be used to evaluate the magnitude of the conformational change associated with Dic binding $[39,44]$ (Figure S5 and Tables S4 and S5). Basically, these two parameters are related with the sum of an intrinsic binding contribution (that can be estimated from the crystal structure of $\mathrm{NQO}_{\text {dic }}$ ) and a second term originating from the conformational change induced upon ligand binding [39]. This second term is straighforwardly converted to the number of residues involved in the conformational change using 
structure-energetic relationships for protein folding (thus yielding the parameter $\mathrm{N}_{\text {conf }}$ ). Dic binding caused a minimal conformational change upon binding to all variants, with $\mathrm{N}_{\text {conf }}$ values (average of the two methods) of 10-20 residues (Figure 6D and Table S6).

3.6. Structural Analysis and Energy Calculations Provide Insight into Mutational Effects on Protein Stability and Function

To provide further molecular insight into the effects of this set of eight mutations on different NQO1 traits, we carried out in silico mutagenesis and structural and energetic analysis in both the holo-forms ( $\left.\mathrm{NQO1}_{\text {holo }}\right)$ and Dic bound forms $\left(\mathrm{NQO}_{\text {dic }}\right)$. An overview of the most relevant structural consequences for each mutation are compiled in Figure 7.

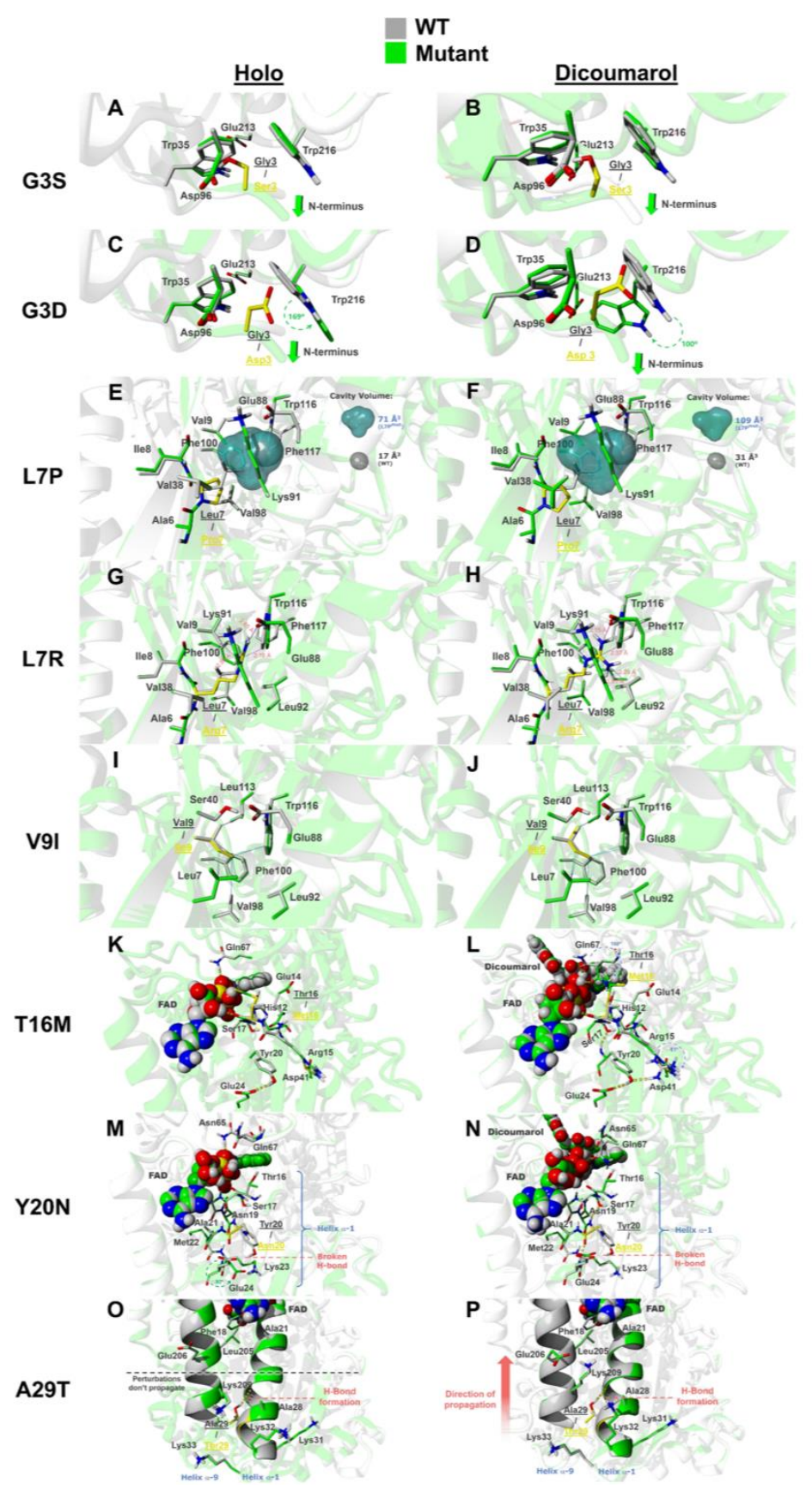

Figure 7. Overview of the mutational effects from in silico mutagenesis. Gray-colored amino acids represent the WT structure while green-colored amino acids represent the mutated models. Mutated 
residues are shown as sticks colored in yellow for each case. $(\mathbf{A}, \mathbf{B}) \mathrm{NQO}_{\text {holo }}(\mathbf{A})$ and $\mathrm{NQO} 1_{\text {dic }}(\mathbf{B})$ states with the G3S mutation. Green arrows indicate displacement of the N-terminus on the opposite side of the mutation. (C,D) $\mathrm{NQO}_{\text {holo }}(\mathbf{C})$ and $\mathrm{NQO}_{\text {dic }}$ (D) states with the G3D mutation. Green dashed arrows indicate side chain rotation. $(\mathbf{E}, \mathbf{F}) \mathrm{NQO}_{\text {holo }}(\mathbf{E})$ and $\mathrm{NQO}_{\text {dic }}(\mathbf{F})$ with the L7P mutation. Blue and gray surfaces represent cavities in the protein core for the mutant and WT, respectively. $(\mathbf{G}, \mathbf{H}) \mathrm{NQO}_{\text {holo }}(\mathbf{G})$ and $\mathrm{NQO}_{\text {dic }}(\mathbf{H})$ with the L7R mutation. Clashes are shown as blue solid lines marked with inter-atomic distances. $(\mathbf{I}, \mathbf{J}) \mathrm{NQO}_{\text {holo }}(\mathbf{I})$ and $\mathrm{NQO} 1_{\text {dic }}(\mathbf{J})$ with the V9I mutation. Hydrophobic interactions are shown as solid lines between interacting atoms colored gray and blue for the interactions created by V9 (WT) and I9 (V9I mutant) residues, respectively. $(\mathbf{K}, \mathbf{L}) \mathrm{NQO}_{\text {holo }}(\mathbf{K})$ and $\mathrm{NQO1}_{\text {dic }}(\mathbf{L})$ with the T16M mutation. Ligand atoms are displayed in ball representation. Hydrogen bonds are depicted as yellow dashed cylinders. $(\mathbf{M}, \mathbf{N}) \mathrm{NQO}_{\text {holo }}(\mathbf{M})$ and $\mathrm{NQO}_{\text {dic }}(\mathbf{N})$ with the Y20N mutation. $(\mathbf{O}, \mathbf{P}) \mathrm{NQO}_{\text {holo }}(\mathbf{O})$ and $\mathrm{NQO}_{\text {dic }}(\mathbf{P})$ with the A29T mutation. Helices $\alpha 1$ and $\alpha 9$ are shown in ribbon representation.

\subsubsection{The Mutations G3S and G3D}

Our experimental analyses have shown that the mutations G3S and G3D are essentially neutral. In the G3S mutant, modeling shows that S3 is surrounded by an hydrophobic patch formed by W35 and W216 and by two negatively charged amino acids, namely D96 and E213, in both NQO1 ${ }_{\text {holo }}$ and $\mathrm{NQO1}_{\text {dic }}$ forms (Figure 7A,B). The mutation G3S promotes local changes in its neighboring amino acids but also displaces the $\mathrm{N}$-terminus of the protein towards the opposite direction of its side-chain, thus helping accommodate the perturbation generated upon the introduction of a bulkier and polar residue. Overall energetic scoring by Rosetta suggested that this mutation has a mild-moderate destabilizing effect on $\mathrm{NQO}_{\text {holo }}$, whereas this effect is mild in the $\mathrm{NQO}_{\text {dic }}$ state (Table 2). The most relevant contributions to this destabilization in both the $\mathrm{NQO}_{\text {holo }}$ and $\mathrm{NQO}_{\text {dic }}$ states seem to arise from (Table S6): (i) unfavorable repulsive energy ( $\Delta$ fa_rep), whose increase accounts for an increment in the number of atoms that come into contact upon mutation, which is in agreement with the mutated residue bumping into other residues, such as W35 and W216, thus affecting the repulsive van der Waals energy; (ii) unfavorable isotropic solvation energy ( $\Delta \mathrm{fa} \_$sol), which arises from burial of polar residues upon mutation (i.e., primarily that of the side chain of S3 that becomes embedded in the hydrophobic patch located around the mutation site).

Table 2. Mutational effects on conformational stability (as folding free energy between the mutant and WT proteins, $\Delta \Delta \mathrm{G}$, in $\mathrm{kcal} \cdot \mathrm{mol}^{-1}$, in the $\mathrm{NQO}_{\text {holo }}$ and $\mathrm{NQO}_{\text {dic }}$ states, determined by Rosseta). Negative values indicate a destabilizing effect whereas positive values indicate a stabilizing effect.

\begin{tabular}{ccccccccc}
\hline & G3S & G3D & L7P & L7R & V9I & T16M & Y20N & A29T \\
\hline NQO1 $_{\text {holo }}$ & -5.0 & -11.6 & -29.4 & -14.1 & 1.9 & -0.8 & -8.1 & -10.9 \\
\hline NQO1 $_{\text {dic }}$ & -1.5 & -10.1 & -25.5 & -35.9 & 0.6 & 0.8 & -5.4 & 0.4 \\
\hline
\end{tabular}

Modeling of the mutation G3D shows larger structural and energetic distortions than those observed for the mutation G3S (Figure 7C,D), predicting moderate-large destabilizing effects in the $\mathrm{NQO1}_{\text {holo }}$ and $\mathrm{NQO}_{\text {dic }}$ states (Table 2). This difference between the mutations G3S and G3D can be easily explained due to nature of the mutations, since G3D introduces a bulkier and charged residue that perturbs the surrounding hydrophobic patch, particularly residues W35 and W216-especially the latter one, which undergoes a $100^{\circ}$ rotation around its $\chi_{1}$ angle in the $\mathrm{NQO}_{\text {dic }}$ state and $169^{\circ}$ around $\chi_{2}$ in the $\mathrm{NQO}_{\text {holo }}$ state. This local conformational change generates a significant penalization due to the increase in the repulsive van der Waals energy and unfavorable isotropic solvation energy terms, presumably due to the insertion of a negatively charged residue into the hydrophobic patch formed by W35 and W216 (an effect seen in both NQO1 $1_{\text {holo }}$ and NQO1 $1_{\text {dic }}$ forms) (Figure 7A-D and Table S6). This may explain why the $\mathrm{N}$-terminus is pushed a few $\AA$ away from the mutated site 
(Figure 7C,D, green arrow shows direction of displacement) in the model of the G3D mutant. This could suggest that the $\mathrm{N}$-terminus of the protein might help to reduce and prevent clashes by potentially accommodating some of the observed perturbations. This also possibly explains the minimal changes in thermal stability and ligand binding observed experimentally, although the mutation is perceived as destabilizing by Rosetta.

\subsubsection{The Mutations L7P and L7R}

Our experimental analyses have indicated that the mutations L7P and L7R are heavily destabilizing (Figure 2). Regarding the mutation L7P, we must note that proline is rarely found in the middle of $\beta$-sheets as its presence is disfavored because of the lack of accessible backbone $\mathrm{N}-\mathrm{H}$ group to participate in hydrogen bonding, thus disrupting the $\beta$-sheet structure $[95,96]$. Energetic scoring by Rosetta analysis supports the tremendous destabilizing effect of the mutation L7P in both $\mathrm{NQO}_{\text {holo }}$ and $\mathrm{NQO}_{\text {dic }}$ states (Table 2). L7 is surrounded by a hydrophobic environment in the core of the protein, composed by A6, I8, V9, V38, L92, V98, F100 and W116 (Figure 7E,F). Most of the hydrophobic interactions established by L7 in this pocket are essentially abolished in the L7P mutant (in both NQO1 $1_{\text {holo }}$ and $\mathrm{NQO1}_{\text {dic }}$ states), likely due to the cavity created by the mutation (Figure 7E,F). In the WT protein, there is only a small cavity with a volume of 17 and $31 \AA^{3}$ (NQO1 ${ }_{\text {holo }}$ and $\mathrm{NQO}_{\text {dic }}$ forms, respectively), whereas the L7P mutation increases the size of this cavity to a volume of 71 and $109 \AA^{3}$ (NQO1 ${ }_{\text {holo }}$ and $\mathrm{NQO1}_{\text {dic }}$ forms, respectively). This cavity in the protein core may be, in part, responsible for the great destabilizing character of the mutation, as reflected in the energetic penalization in the attractive energy (fa_atr) term (Table S6). Interestingly, parametrizations of the conformational destabilization caused by cavities in model proteins have provided values in the range of $24-36 \mathrm{cal} \cdot \mathrm{mol}^{-1} \cdot \AA^{3}[97,98]$, thus allowing to calculate that just this cavity (without considering additional perturbations caused) would destabilize the native structure by $1.3-1.9 \mathrm{kcal} \cdot \mathrm{mol}^{-1}\left(\mathrm{NQO}_{\text {holo }}\right)$ and $1.9-2.8 \mathrm{kcal} \cdot \mathrm{mol}^{-1}\left(\mathrm{NQO}_{\mathrm{dic}}\right)$. Additionally, in both $\mathrm{NQO}_{\text {holo }}$ and $\mathrm{NQO}_{\text {dic }}$ states, the mutation L7P breaks a backbone hydrogen bond between $\beta 1$ and $\beta 3$ parallel strands that destabilizes the $\beta$-sheet structure (Figure 8B,E), thus reducing the long-range hydrogen bonds energy term (hbond_lr_bb) (Table S6). The perturbation generated by P7 is accentuated by the distortion in dihedral $\Phi$ and $\Psi$ angles caused by the geometry of the mutated residue and the prevention of hydrogen bond formation in the proximity of the mutated site (Figure $8 \mathrm{C}, \mathrm{F}$ ).

The mutation L7R is also highly destabilizing, as calculated by Rosetta (Table 2). This mutation introduces a severely perturbing, positively charged residue in the formerly described well-packed hydrophobic pocket (Figure 7G,H). Unlike L7P, L7R does not generate a cavity (Figure 7E,F) but instead causes steric clashes with neighbouring residues (Figure 7G,H and Table S7) in both $\mathrm{NQO}_{\text {holo }}$ and $\mathrm{NQO}_{\text {dic }}$ states. In this case, conformational destabilization mainly arises from unfavorable isotropic solvation energy ( $\Delta$ fa_sol) (Table S6), likely due to the burial of a charged residue in a hydrophobic environment. 

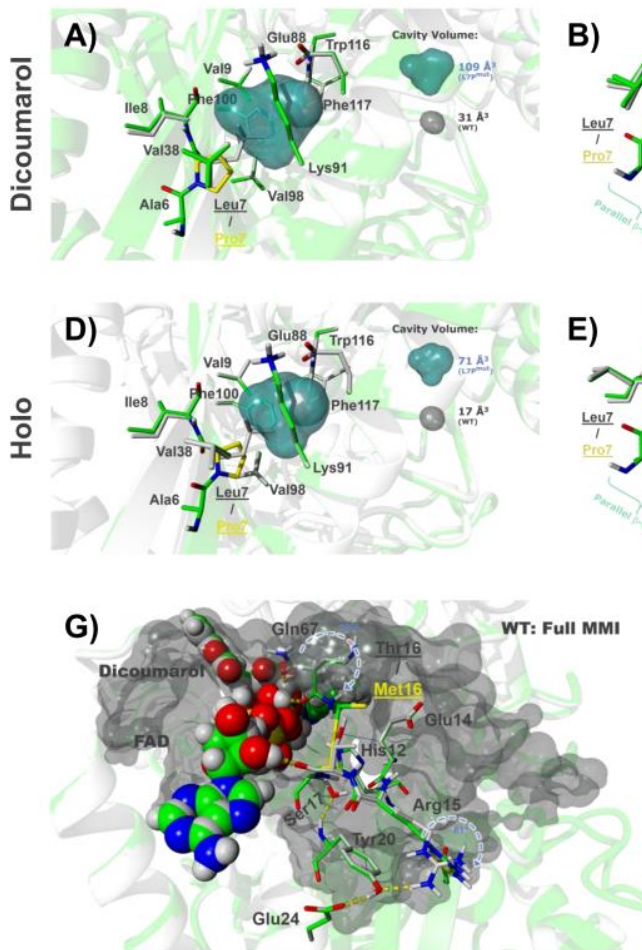

I)

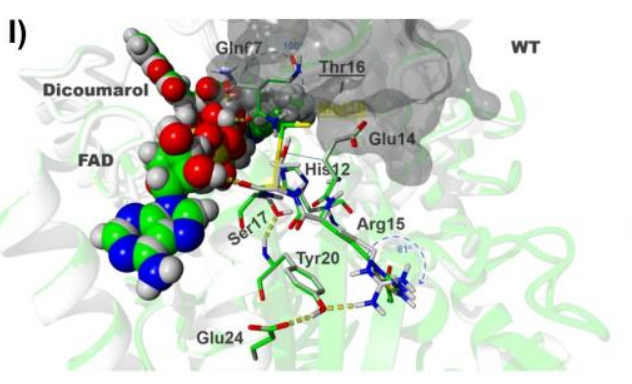

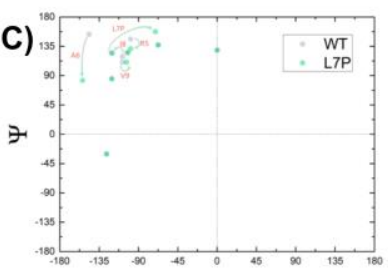
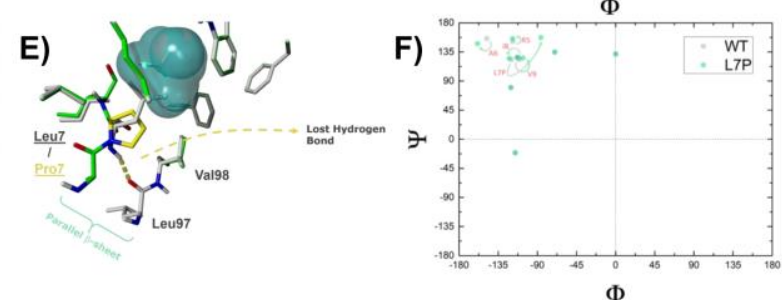

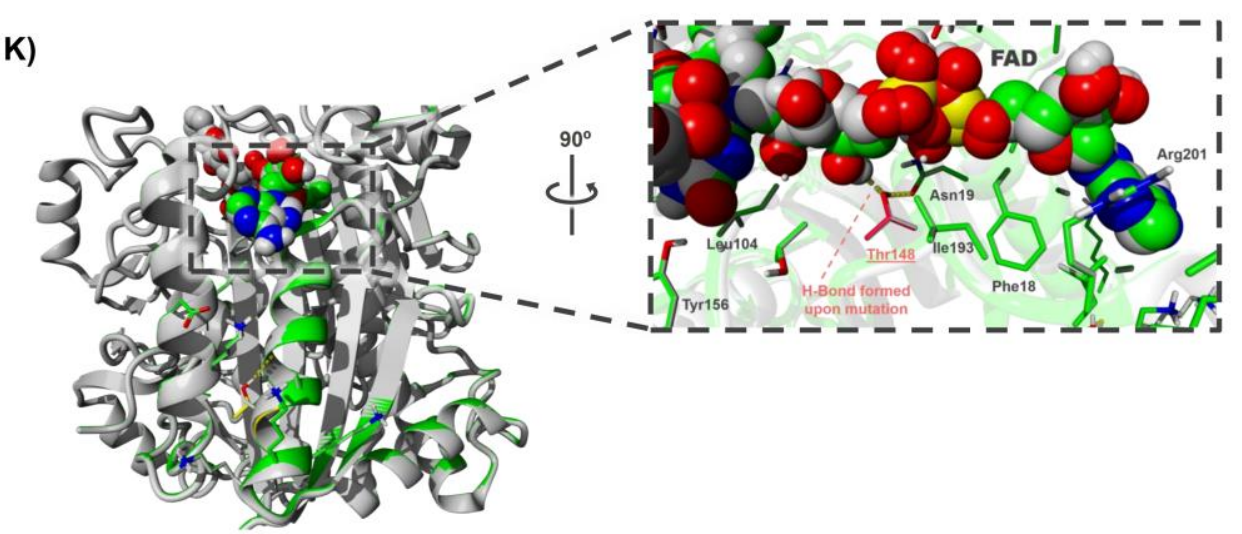

Figure 8. Detailed structural analysis of the mutations L7P (A-F), T16M (G-J) and A29T (K). (A) Overall view of the L7P site in the NQO1 $1_{\text {dic }}$ state (mutated residue in yellow). The mutation generates a cavity in the hydrophobic core of the protein (semi-transparent blue for L7P and gray for WT protein). (B) Close-up view on the hydrogen bond disrupted by L7P in the $\mathrm{NQO1}_{\text {dic }}$ state. (C) Ramachandran plot of the first ten amino acids in the $\mathrm{NQO}_{\text {dic }}$ state. Arrows indicate the displacement in dihedral angles caused by L7P mutation that affects the $\beta$-sheet pattern near the mutated site. (D) Close-up of the mutated site (in yellow) in the $\mathrm{NQO}_{\text {holo }}$ state. The mutation L7P generates a cavity in the hydrophobic core of the protein represented in semi-transparent blue (L7P) and gray (WT). (E) Zoom-in 
on the hydrogen bond formerly established by L7 and disrupted by the L7P mutation in NQO1 $1_{\text {holo }}$. (F) Ramachandran plot of the first ten amino acids in $\mathrm{NQO}_{\text {holo }}$ showing, with arrows, the displacement caused by L7P mutation that affects the $\beta$-sheet pattern near the mutated site. (G-J) Effects of the T16M mutation on the MMI of the NQO1 $1_{\text {dic }}$ state. The MMI surface is shown in gray for the WT and in bluish for the T16M mutant. The overall view is similar to that shown in Figure 7L. The complete MMI for the WT protein $(\mathbf{G})$ vs. T16M mutant $(\mathbf{H})$ is shown. Mutational effects are found in the top right corner. $(\mathbf{I}, \mathbf{J})$ Similar representations to those in panels $(\mathbf{G}, \mathbf{H})$ but only showing residues of the MMI close $(<5 \AA)$ to the mutated site. (K) Close-up view of the hydrogen bond formed between FAD and residue T148 in the A29T mutant in the $\mathrm{NQO}_{\text {dic }}$ state.

\subsubsection{The Mutation V9I}

Experimental characterization of the mutation V9I has shown nearly neutral effects. As analyzed by Rosetta, this mutation has marginal effects on conformational stability (Table 2). V9 (in NQO1 WT) and I9 (in the V9I mutant) are embedded in a similar hydrophobic environment to that of L7 (that is perturbed by the mutations L7P and L7R) (Figure 7I,J). Interestingly, the mutation V9I causes a favorable change in the van der Waals energy (fa_atr) term (Table S6) indicating increased van der Waals interactions with neighbouring hydrophobic residues (Figure 7I,J and Table S8) in both NQO1 $1_{\text {holo }}$ and $\mathrm{NQO}_{\text {dic }}$ states. Even though the introduction of a larger amino acid causes some structural strain (i.e., slightly displaces some neighbouring amino acids), this effect is essentially canceled out by the gain in favorable van der Waals interactions (Table S8).

\subsubsection{The Mutation T16M}

Our experimental analyses have shown that T16M has phenotypic consequences in several traits, particularly FAD binding, thermal stability and local stability of the TCS. Analysis by Rosetta shows that T16M mutation should have small effects on conformational stability (Table 2). Interestingly, decomposition of energetic contributions provided by Rosetta (Table S6) indicates different and opposing energetic contributions that nearly cancel out. A detailed structural analysis may provide further insight into these effects as well as in the phenotypic consequences of this mutation (Figure $7 \mathrm{~K}, \mathrm{~L}$ ).

The mutation $\mathrm{T} 16 \mathrm{M}$ produces mild perturbations of the $\mathrm{NQO}_{1}$ holo state (Figure $7 \mathrm{~K}$ ), causing small side-chain adjustments affecting residues R15, Y20, E24 and N65. Y20 and E24 residues are located in helix $\alpha 1$ and their rearrangement, together with the presence of M16, produces a small displacement in the FAD binding pose that could explain the effect of T16M on FAD binding.

Interestingly, we also observed noticeable changes in the modeled structures of $\mathrm{NQO}_{\text {dic }}$. The residue M16 (located in the first shell of interaction with the ligand FAD at a shortest distance $<5 \AA$ ) interacts through hydrophobic contacts with the residues nearby, such as the aliphatic portion of E14 side chain, and brings much closer the residue Q67. This, together with the perturbation in the backbone atoms of the residues adjacent to the mutation site, generates a moderate change in conformation on some residues close to the ligand binding site, ultimately altering its initial pose (i.e., that present in the WT). The residues affected by direct or indirect interaction with M16 are H12, E14, M16 (backbone) and F18, but the amino acids suffering the largest conformational changes on their side chains are Q67 and $\mathrm{R} 15\left(100^{\circ}\right.$ and $61^{\circ}$ rotation across $\chi_{2}$ and $\chi_{3}$ angles, respectively), whose motions appear to distort important interactions for ligand binding (see Figure 7L and Video S1). The most significant change results in the reorientation of Q67, which now faces M16 side chain and, while it maintains its original hydrogen bond with FAD, forms another hydrogen bond with the carbonyl group present in F66 residue. The other large conformational change involves R15, which was engaged (in the WT NQO1 $1_{\text {dic }}$ state) in a hydrogen bond network with residues Y20 and E24 (located in helix $\alpha 1$ ). The mutation T16M disrupts the hydrogen bond with Y20 and makes R15 form two hydrogen bonds with D41 (achieving a virtually similar conformation to that shown in the WT NQO1 holo state, see Video S2). It is interesting to speculate that since Dic is a competitive inhibitor of both the NAD $(\mathrm{P}) \mathrm{H}$ and the substrate, these structural alterations may have consequences on the NQO1 catalytic cycle. 
The residue M16 (as well as the wild-type residue T16) is part of the MMI. In fact, the changes described above can also be translated to some alterations of the MMI (Figure 8G-J) and may explain the effects of T16M in both thermal stability and FAD binding. The adjustments and side-chain reorientations described earlier are summarized in Video S1 (morphing conformations between the WT and T16M as NQO1 $1_{\text {dic }}$ ) and should be compared to Video S2 (morphing conformations between WT NQO1 $1_{\text {dic }}$ and $\mathrm{NQO}_{\text {holo }}$ states of the protein).

\subsubsection{The Mutation Y20N}

The mutant Y20N shows noticeable effects on thermal stability and FAD binding. Stability calculations using Rosetta provide moderate destabilizing effects (Table 2). Inspection of the modeled structures revealed certain structural alterations caused by this mutation (Figure $7 \mathrm{M}, \mathrm{N}$ ). Introduction of the smaller $\mathrm{N} 20$ residue prevents the formation of hydrogen bonds (in both $\mathrm{NQO}_{\text {holo }}$ and $\mathrm{NQO}_{\text {dic }}$ states) observed between Y20 and residues E24 and R15 (note that the latter residues also played important roles in the structural alterations caused by the mutation T16M). This alteration in hydrogen bonding causes slight backbone and side-chain movements on the residues nearby that are also part of the $\alpha 1$ helix, which also played a noteworthy role in the alterations experienced upon T16M mutation. These adjustments in the $\mathrm{NQO}_{\text {dic }}$ state cause slight changes in FAD binding pose (Video S3), comparable to those observed for the T16M mutation. In the holo form of the protein, a similar situation occurs when Y20 residue is mutated to N20. The broken hydrogen bond network destabilizes the surroundings of the mutated site, ultimately altering ligand binding pose (Video S4). In this case, E24 also rotates, by $\sim 92^{\circ}$, its side chain around the $\chi_{2}$ angle, presumably due to the inability to establish a hydrogen bond with residue N20. Overall, these results provide structural insight into the effect of Y20N on FAD binding affinity and also predict that this mutation might also affect the catalytic cycle of NQO1.

\subsubsection{The Mutation A29T}

The mutation A29T has essentially neutral effects, except for a moderate decrease in FAD binding affinity. Stability calculations using Rossetta suggest that this mutation substantially destabilizes the $\mathrm{NQO}_{\text {holo }}$ state (Table 2). Structural modeling shows that the T29 in this mutant establishes an intra-helix hydrogen bond between its side chain hydroxyl group and the carbonyl backbone group of amino acid $\mathrm{A} 25$ in both $\mathrm{NQO}_{\text {holo }}$ and $\mathrm{NQO}_{\text {dic }}$ states (Figure 7O,P), which might stabilize helix $\alpha 1$. In the $\mathrm{NQO}_{\text {holo }}$ form, this mutation causes side-chain conformational regroupings in the vicinity of the mutated site. However, in the $\mathrm{NQO}_{\text {dic }}$ state, these local adjustments propagate through helices $\alpha 1$ and $\alpha 9$ towards the FAD binding site and, ultimately, slightly modify its binding mode (See Videos S5 and S6) by forming a hydrogen bond with $\mathrm{O} \gamma 1$ from residue T148 in the FAD binding site (Figure $8 \mathrm{~K}$ ). This interaction might contribute to the destabilizing effect of this mutation when the inhibitor is bound (as calculated by Rosetta) (Table 2).

\subsection{The Role of Protein Local Dynamics and Stability on Mutational Effects}

Protein structural dynamics are, likely, critical to understand many features of NQO1, such as the stabilizing effect of FAD binding towards proteasomal degradation of WT NQO1, ligand binding-mediated changes in the interaction with other proteins and the functional and stability alterations caused by missense mutations and polymorphisms [19,39,41,52,53,90,91]. Recently, we used hydrogen/deuterium exchange (HDX) monitored by mass spectrometry to provide a high-resolution map of the changes in protein local dynamics and stability of WT NQO1 in different ligation states $\left(\mathrm{NQO1}_{\mathrm{apo}}, \mathrm{NQO}_{\text {holo }}\right.$ and $\left.\mathrm{NQO}_{\text {dic }}\right)$ [90]. This study has allowed identification of a stable core (as non-exchanging segments) in $\mathrm{NQO}_{\text {apo }}$ that holds the NQO1 dimer, as well as the short- and long-range propagation of ligand binding effects (i.e., FAD and Dic). We have, thus, used this dynamic information in different ligation states to provide further molecular insight into the mutational effects in different protein traits experimentally characterized in this work. 
Residues L7 and V9 belong to regions with very high structural stability in the NQO1 apo state (i.e., these are located in the stable core) (Figure 9 and Figure S6 and Table S9). It is plausible that a significantly large structural perturbation at these sites would have dramatic effects on the ability of NQO1 to fold or the stability of the folded protein. Consequently, we could not obtain stable and soluble NQO1 protein with the largely-disrupting L7P or L7R mutations (Figure 2), whereas the more conservative V9I mutation mildly decreased dimer stability (Figure 3). Residues G3 and A29 are located in regions with moderate structural stability and their stability is hardly sensitive to ligand binding (Figure 9 and Figure S6, Tables S9 and S10). Accordingly, mutations G3S, G3D and A29T have mild effects on protein stability (Figure 3) and ligand binding, except for the six-fold decrease in FAD binding affinity in A29T (Figure 6). Residues T16 and Y20 are found in regions with low structural stability in $\mathrm{NQO1}_{\mathrm{apo}}$, although these regions manifest large structural stabilization by the stepwise binding of FAD and Dic (Figure 9 and Figure S6, Tables S9 and S10). Therefore, these analyses help to explain that mutations T16M and Y20N have moderate effects on protein stability (Figure 3) but exhibit important alterations in FAD binding (Figure 6), particularly the mutation T16M that affects a residue in close contact with the FAD molecule.
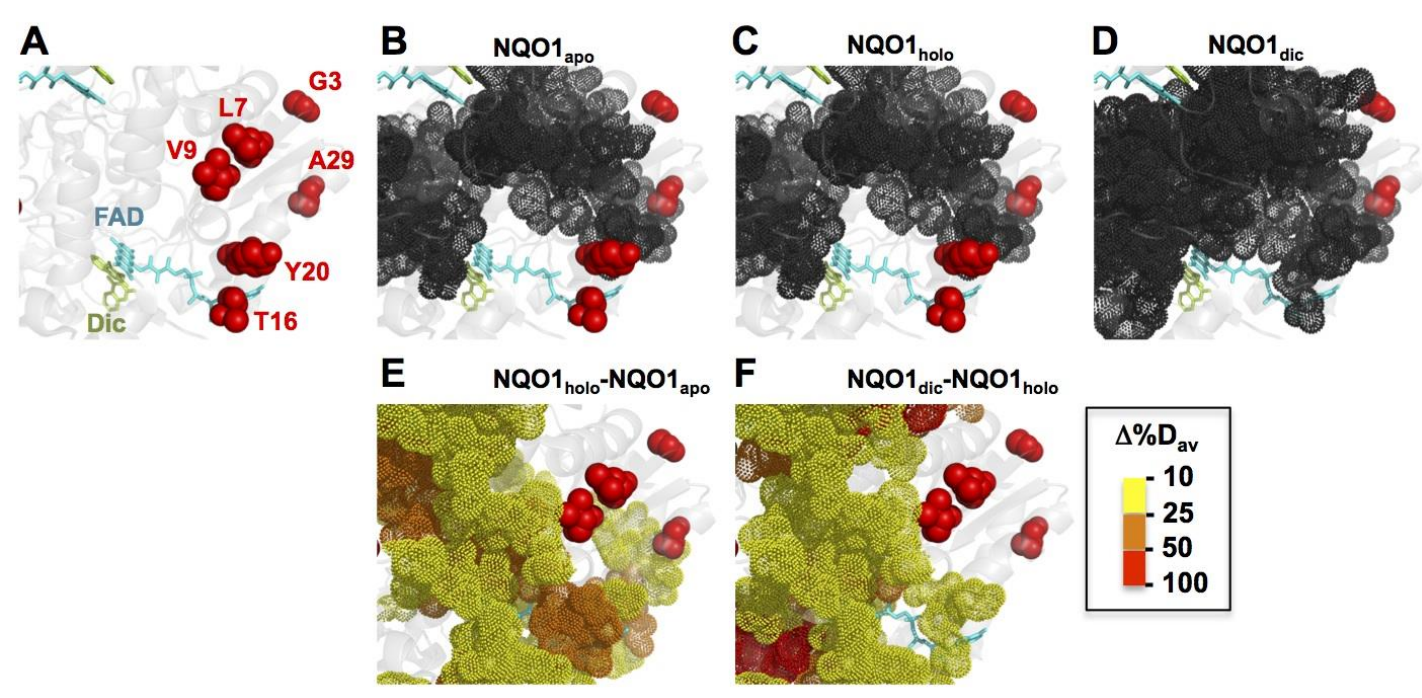

Figure 9. Location of mutated residues regarding local stability measurements by hydrogen/deuterium exchange (HDX). (A) Structural location of mutated residues regarding bound FAD and Dic. (B-D) Residues in dark grey/dot representation indicate those regions of the WT protein (NQO1 apo, $\mathrm{NQO}_{\text {holo }}$ and $\left.\mathrm{NQO}_{\text {dic }}\right)$ that are highly stable $(<20 \%$ HDX after $3 \mathrm{~h})$. (E,F) Residues in dot representation (see color scale) indicate those whose stability (as $\Delta \% \mathrm{D}_{\mathrm{av}}$ ) was increased between the two states $\left(\mathrm{NQO}_{1}\right.$ holo vs. $\mathrm{NQO}_{\mathrm{apo}}$ and $\mathrm{NQO} 1_{\text {dic }}$ vs. $\left.\mathrm{NQO} 1_{\text {holo }}\right)$. Plots are generated using primary data from [90].

Inspection of those regions of the protein that show high stability and/or strong ligand binding-dependent stability may provide further explananation for some of the functional alterations displayed by mutant NQO1 proteins. In Figure 9A-D, we show the highly stable regions (with minimal HDX after $3 \mathrm{~h}$ ) in $\mathrm{NQO}_{\text {apo, }} \mathrm{NQO}_{\text {holo }}$ and $\mathrm{NQO1}_{\text {dic }}$ (see also Table S9). Residues L7 and V9 belong to this stable core in any of the three ligation states, whereas G3 does not in any of these ligation states (Figure 9A-D). The residue A29 is adjacent to this core in all three ligation states. In Figure 9E,F, we show those protein regions that undergo large stability changes upon binding FAD (Figure 9E) and Dic (Figure 9F). Regarding FAD binding, residues T16 and Y20 are buried in these regions that undergo large stability changes upon binding, thus supporting that their effects might be associated with alterations of the structural stabilization provided by FAD binding. A similar interpretation can be proposed to explain the effects of the mutation A29T, which affects a residue adjacent to those regions largely stabilized by FAD binding. Consequently, the lower affinity for FAD in the mutants T16M, 
Y20N and A29T could be partially explained by their perturbing effects on regions stabilized upon FAD binding (Figure 9E). A similar interpretation could apply for the lack of effect of the mutations studied in this work on Dic binding, since overall, the residues affected by these mutations are located in regions that are not largely stabilized upon inhibitor binding (Figure 9F).

\subsection{Correlations between Loss-of-Function Scores Derived from Experimental Analysis of Mutational Effects} and Bioinformatic Tools

So far, we have shown that naturally-occurring mutations, found in either targeted (i.e., disease-associated) samples (COSMIC database) or whole-genome sequencing initiatives (gnomAD database), can affect, to different extents, diverse features in a multi-functional protein, such as NQO1. These results (compiled in the so-called $N_{t}$ set) extend our previous knowledge on a variety of natural (cancer-associated, P187S and K240Q) as well as non-natural mutations generated at the P187 and K240 sites (named as P187 and K240 sets; see Table 2) [19,39]. To what extent can we predict the potential molecular and functional consequences (plausibly linked to their potential pathogenicity) of this set of 22 mutations using a combination of some of the most common bioinformatic algorithms (Table 4)? This task is challenging, since current algorithms usually provide a single metric to assess this potential pathogenicity, whereas, as we have shown here, mutational effects on protein functionality are intrinsically complex (i.e., the protein is multi-functional). In an attempt to assess our capacity to establish correlations between experimental analysis and bioinformatic predictive tools, we have determined a simple experimental score (ES) for this set of 22 mutations that takes into account, in a semi-quantitative manner, their effects on different functional features (Table 2). Although these are features characterized in vitro, we may expect that some of them can reflect loss-of-function phenotypes in vivo (and, thus, potential pathogenicity due to loss-of-function). For instance, severe folding/solubility problems, impaired thermal stability and reduced local stability are likely associated with folding and stability problems inside cells (e.g., the consequences of P187S on these features are clearly associated with its intracellular stability; $[41,43,52,68]$ ). In addition, reduced local stability and FAD binding affinity can be also associated with low intracellular activity and stability due to increased population of the inactive and degradation-prone apo-state (e.g., in the phosphomimetic mutant S82D; [91]). Reduced affinity for the inhibitor Dic may also reflect conformational alterations associated with enhanced intracellular degradation (the P187S polymorphism; [41]) and/or reduced intracellular sensitivity towards NQO1 inhibition, leading to reduced cancer growth $[39,44,63,93]$.

ESs were meant to provide a semiquantitative ranking of pathogeniticy: an ES of 1 corresponded to a severe mutation, ES of 2 to a mild one and ES of 3 to a neutral mutation. Calculation of the ESs for this set of mutants showed that five mutations caused large defects on protein stability and/or solubility, and thus, these were considered as severe (i.e., ES of 1), including the mutations L7P and L7R (Table 2). The remaining 17 mutations showed an ES ranging from 1.4-3.0. Among the lowest ESs, we found the polymorphism P187S, the gold standard of loss-of-function in NQO1 [41,43,52,68]. Most of the mutations in the $\mathrm{N}_{\mathrm{t}}$ and K240 sets showed mild to neutral ESs (ES from 2.2 to 3), whereas variants in the P187 set were more deleterious (ES from 1 to 2).

We used these ESs to ascertain whether popular bioinformatic tools would predict and rank potential pathogenic effects. To this end, we generated bioinformatic scores (BSs) to be semiquantitatively compared with the ESs (Tables 3 and 4). The results from these correlations are shown in Figure 10. When the full mutant set was analyzed, we observed a significant positive correlation between ESs and BSs (Figure 10). Visual inspection of this plot shows this correlation performs differently for different sets of mutations. The correlation for the $\mathrm{N}_{\mathrm{t}}$ set is much stronger than that for the P187 set and, particularly, than that for the K240 set (Figure 10). The origin of this difference in performance is unclear. However, the very poor perfomance with the K240 set might have a straightforward explanation: this residue is highly conserved among mammalian NQO1 sequences, thus likely explaining that bioinformatic tools may identify mutations in this residue as potentially pathogenic. However, experimental analyses have shown that even highly disrupting 
mutants (such as K240E and K240G) still show many functional aspects not departing much from those of WT NQO1 [19,39]. These results were somehow expected: current bioinformatic approaches may provide some good overall results for genotype-phenotype correlations but fail to predict accurately and individually this correlation for individual mutations (one of the holy grails of personalized medicine, [99]), particularly when mutations affect highly conserved residues.

Table 3. Classification of phenotypic consequences due to NQO1 mutations based on experimental data. The experimental scores (ES) are calculated as the average from individual scores determined for each phenotypic trait as follows: Expression:,$+++ 50-100 \%$ of WT levels;,$++ 20-50 \%$ of WT levels; ,$+<20 \%$ of WT levels; Thermal stability: +++ , within $2{ }^{\circ} \mathrm{C}$ of WT $\mathrm{T}_{\mathrm{m}} ;++$, within $2-5{ }^{\circ} \mathrm{C}$ of $\mathrm{WT} \mathrm{T}_{\mathrm{m}} ;+$, $>5^{\circ} \mathrm{C}$ lower than WT T ; Proteolysis: +++ , rate constant within three-fold vs. WT $\left(0.65 \mathrm{kcal} \cdot \mathrm{mol}^{-1}\right) ;++$, within 3 to 30 -fold vs. WT $\left(2 \mathrm{kcal} \cdot \mathrm{mol}^{-1}\right)$; $++>30$-fold faster than WT (over $\left.2 \mathrm{kcal} \cdot \mathrm{mol}^{-1}\right)$; FAD binding: +++ , within three-fold of WT $K_{\mathrm{d}} ;++$, within 3-10 fold higher than WT $K_{\mathrm{d}} ;+,>10$-fold higher than WT $K_{\mathrm{d}}$; Dic binding: +++ , within three-fold of WT $K_{\mathrm{d}} ;++$, within 3-10 fold higher than WT $K_{\mathrm{d}} ;+,>10$-fold higher than WT $K_{\mathrm{d}}$.

\begin{tabular}{|c|c|c|c|c|c|c|}
\hline Variant & Expression & Thermal Stability & Proteolysis & FAD Binding & Dic Binding & ES \\
\hline G3S & +++ & +++ & +++ & +++ & +++ & 3 \\
\hline G3D & +++ & +++ & +++ & +++ & +++ & 3 \\
\hline L7P & + & & & & & 1 \\
\hline L7R & + & & & & & 1 \\
\hline V9I & +++ & +++ & +++ & +++ & +++ & 3 \\
\hline $\mathrm{T} 16 \mathrm{M}$ & +++ & ++ & ++ & + & +++ & 2.2 \\
\hline Y20N & +++ & ++ & +++ & ++ & +++ & 2.6 \\
\hline A29T & +++ & +++ & +++ & ++ & +++ & 2.8 \\
\hline P187S & +++ & + & + & + & + & 1.4 \\
\hline P187E & + & & & & & 1 \\
\hline P187R & + & & & & & 1 \\
\hline P187L & + & & & & & 1 \\
\hline P187A & +++ & ++ & + & ++ & ++ & 2 \\
\hline P187G & ++ & + & ++ & +++ & ++ & 2 \\
\hline $\mathrm{P} 187 \mathrm{~T}$ & +++ & ++ & + & ++ & + & 1.8 \\
\hline K240Q & +++ & +++ & ++ & ++ & ++ & 2.4 \\
\hline K240I & +++ & +++ & ++ & +++ & +++ & 2.8 \\
\hline K240E & +++ & +++ & + & ++ & ++ & 2.2 \\
\hline K240T & +++ & +++ & +++ & ++ & +++ & 2.8 \\
\hline $\mathrm{K} 240 \mathrm{H}$ & +++ & +++ & ++ & ++ & +++ & 2.6 \\
\hline K240A & +++ & +++ & + & +++ & +++ & 2.6 \\
\hline K240G & +++ & +++ & + & ++ & ++ & 2.2 \\
\hline
\end{tabular}


Table 4. Classification of phenotypic consequences of NQO1 mutations based on bioinformatic analysis. The bioinformatic scores (BSs) are calculated as the average from individual scores provided by different algorithms as follows: Polyphen-2: +++ , Benign; ++ , Possibly damaging; + , Probably damaging; SIFT: +++ , tolerated; +, Not tolerated; Mutation Taster: +++ , polymorphism; + , disease-causing; Provean: +++ , neutral; + , deleterious; PON-P2: +++ , neutral; ++ , unknown; + , pathogenic. ${ }^{*}$ N.Det.- not determined because two nucleotide have to change.

\begin{tabular}{|c|c|c|c|c|c|c|}
\hline Variant & PolyPhen-2 & SIFT & Mutation Taster & Provean & PON-P2 & BS \\
\hline G3S & +++ & +++ & +++ & +++ & +++ & 3 \\
\hline G3D & +++ & +++ & +++ & +++ & ++ & 2.8 \\
\hline L7P & + & + & + & + & + & 1 \\
\hline L7R & + & + & + & + & + & 1 \\
\hline V9I & +++ & ++ & + & +++ & ++ & 2.2 \\
\hline $\mathrm{T} 16 \mathrm{M}$ & + & + & + & +++ & + & 1.4 \\
\hline Y20N & ++ & +++ & + & +++ & ++ & 2.2 \\
\hline $\mathrm{A} 29 \mathrm{~T}$ & +++ & +++ & + & +++ & +++ & 2.6 \\
\hline P187S & +++ & +++ & +++ & + & ++ & 2 \\
\hline P187E & ++ & + & N.Det. * & + & + & 1.25 \\
\hline P187R & + & + & + & + & + & 1 \\
\hline P187L & + & + & + & + & + & 1 \\
\hline P187A & ++ & +++ & + & + & ++ & 1.8 \\
\hline P187G & ++ & + & N.Det. * & + & + & 1.25 \\
\hline P187T & ++ & +++ & + & + & + & 1.6 \\
\hline K240Q & + & + & + & +++ & ++ & 1.6 \\
\hline K240I & + & + & + & + & ++ & 1.2 \\
\hline $\mathrm{K} 240 \mathrm{E}$ & + & + & + & +++ & ++ & 1.6 \\
\hline $\mathrm{K} 240 \mathrm{~T}$ & + & + & + & +++ & ++ & 1.6 \\
\hline $\mathrm{K} 240 \mathrm{H}$ & + & + & N.Det. * & +++ & ++ & 1.75 \\
\hline $\mathrm{K} 240 \mathrm{~A}$ & + & + & N.Det. * & +++ & ++ & 1.75 \\
\hline K240G & + & + & N.Det. * & + & ++ & 1.25 \\
\hline
\end{tabular}

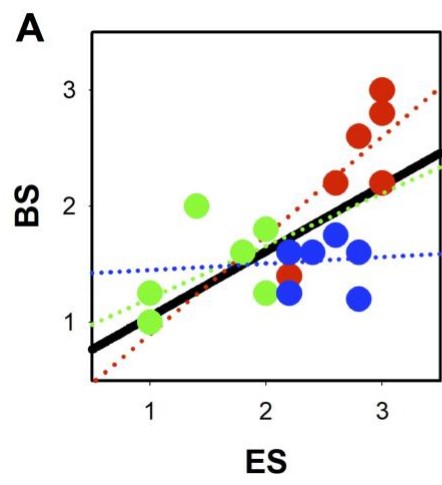

B

$\begin{array}{ccccc} & \text { All } & \mathrm{N}_{\mathrm{t}} \text { set } & \mathrm{P} 187 \text { set } & \mathrm{K} 240 \text { set } \\ \mathbf{R}^{2} & 0.50 & 0.85 & 0.30 & <0.01 \\ \text { intercept } & \begin{array}{c}0.49 \pm 0.28 \\ (p=0.1)\end{array} & \begin{array}{c}0.05 \pm 0.36 \\ (p=0.9)\end{array} & \begin{array}{c}0.76 \pm 0.47 \\ (p=0.17)\end{array} & \begin{array}{c}1.4 \pm 1.0 \\ (p=0.22)\end{array} \\ \text { slope } & \begin{array}{c}0.56 \pm 0.13 \\ \left(p=2 \cdot 10^{-4}\right)\end{array} & \begin{array}{c}0.85 \pm 0.15 \\ \left(p=1.2 \cdot 10^{-3}\right)\end{array} & \begin{array}{c}(p=0.45 \pm 0.31 \\ (p=0.21)\end{array} & \begin{array}{c}0.06 \pm 0.39 \\ (p=0.89)\end{array}\end{array}$

Figure 10. Correlation between experimental scores (ESs) and bioinformatic scores (BSs). (A) Plot of ES vs. BS for all mutants compiled in Tables 3 and 4 . Red circles show mutants belonging to the $N t$ set (the eight mutants characterized in this work) and green and blue circles show mutants belonging to mutations at the P187 and K240 sites, respectively (P187 and K240 sets, see Tables 3 and 4 [39]). The thick black line is the linear regression of all mutants, while dotted lines are linear fittings using one of the three sets $\left(\mathrm{N}_{\mathrm{t}}, \mathrm{P} 187\right.$ or K240 sets; red, green and blue lines, respectively). (B) Best-fit parameters for linear regressions are shown in panel $\mathrm{B}$. 


\section{Conclusions}

From the experimental characterization of the effects of eight naturally-occurring mutations in the multifunctional NQO1 protein, combined with detailed structural analysis and bioinformatic predictions, we can draw several important conclusions and propose future research lines.

First, by investigating the consequences of missense mutations found in whole-genome sequencing studies (with different allelic frequencies; see Table 1) as well as those found in cancer cell lines, we have observed that the severity of the pathogenic effects does not correlate well with their presence in cancer samples (i.e., the mutations L7P and L7R cause similarly catastrophic effects) nor with frequency in untargeted whole-genome sequencing studies (e.g., L7R, a severe mutation, has a 6.5-fold higher allelic frequency than G3S, a neutral mutation). Interestingly, one of the most deleterious natural missense variants in NQO1 (P187S) is also the most common in the human population (with an allelic frequency over 0.3 ) and is the missense variant most robustly associated with predisposition to disease $[47,65]$. These results may apparently contradict recent large-scale mutational analysis in several disease-associated protein systems that showed a negative correlation between allelic frequency of missense mutations and their effects on structural and interacellular stability $[5,9,10,26]$. This might imply that the correlation between allelic frequency and potential pathogenicity is not universal, that NQO1 is just an odd exception or that incorporating multiple functional features (stability, activity, regulation, ... ) into the picture may lead to more complex correlations. Obviously, understanding these and other possible scenarios deserves further research, including the analysis of larger sets of naturally-occuring missense mutations in diverse disease-associated proteins.

Second, structural-energetic-functional relationships of mutational effects are complex, and thus, an integrated view from biochemical and biophysical functional and stability studies, structural modeling and energetic considerations provides a suitable approach to characterize mutational effects in multifunctional proteins. Ideally, this should be also complemented with expression studies and stability analyses in eukaryotic cells. Obviously, all of this cannot be done at a whole-genome scale. However, at a smaller scale, these studies could provide insight into key concepts to be implemented in current in silico approaches for large-scale genotype-phenotype predictions. For instance, it is particularly relevant to consider the locally destabilizing effects of mutations and how these effects can propagate to distant sites in protein structures, thus contributing to pleiotropic (i.e., mulfunctional) effects of missense mutations [19,22,27-29,39,100]. It is also of interest that the threshold to cause intracellular destabilization with pathogenic consequences seems to be quite low, in the order of 2-3 kcal. $\mathrm{mol}^{-1}$ (often determined as the effects on overall conformational stability from experimental or computational methods) $[9,10,26]$. We should also consider local stability effects, not only for mutational effects on intracellular stability but also for functional consequences. Although P187S is known to cause devastating effects on intracellular stability and function, these effects are associated with changes in local stability in the order of $2-3 \mathrm{kcal} \cdot \mathrm{mol}^{-1}$ [41], and effects within this range are observed for other naturally-occurring variants (such as T16M, Y20N, A29T and K240Q) (Table 3). Since propagation of mutations effects to distant sites in protein structure is likely a universal behavior [31,101-103], we would anticipate that the pleitropic effects described for missense mutations in NQO1 could apply to many other disease-associated protein systems [19].

Third, it is also of interest that scores based on quantitative analysis of mutational effects and those built using diverse current bioinformatic tools provided a positive correlation for a set of 22 mutations on NQO1. However, as we have shown, this predictive power is still disappointing when genotype-phenotype correlations are attempted for single mutations and also performs quite poorly for certain mutated sites (e.g., the K240 site). Thus, our work further supports the notion that detailed characterization of mutational effects by extensive functional and stability analysis will help to improve our capacity for accurate and large-scale genotype-phenotype correlations.

Supplementary Materials: The following are available online at http://www.mdpi.com/2075-4426/10/4/207/s1. Figure S1. Representative SDS-PAGE for proteolysis kinetics by thermolysin. Figure S2. Kinetics of proteolysis of NQO1 proteins with thermolysin. Figure S3. Titrations of apo-proteins with FAD. Figure S4. Titrations of NQO1 
variants with Dic at $25^{\circ} \mathrm{C}$. Figure S5. Thermodynamic dissection of Dic binding to NQO1 variants. Figure S6. HDX analysis of structural regions in which NQO1 mutations are localized. Table S1. Effect on conformational stability due to NQO1 mutations determined using structure-based methods. Table S2. Second-order rate constants $\left(k_{\text {prot }}\right)$ for proteolysis by thermolysin and mutational effects on local stability $\left(\Delta \Delta G_{\text {prot }}\right)$. Table S3. FAD binding affinity of NQO1 variants. Table S4. Thermodynamic parameters for Dic binding to NQO1 variants. Table S5. Magnitude of the conformational change $\left(\mathrm{N}_{\text {conf }}\right)$ associated with Dic binding from analysis of apparent binding enthalpies $(\Delta \mathrm{H})$ and heat capacities $\left(\Delta \mathrm{C}_{\mathrm{p}}\right)$. Table S6. Energetic dissection of mutational effects as determined by Rosetta. Table S7. Analysis of steric clashes present in the $\mathrm{NQO}_{\text {holo }}$ and $\mathrm{NQO}_{\mathrm{dic}}$ states caused by the mutation L7P. Table S8. Hydrophobic interactions established between V9 (WT) and I9 (mutant V9I) in the NQO1 $1_{\text {holo }}$ and NQO1 $1_{\text {dic }}$ states. Table S9. Regions with $<20 \%$ HDX after $3 \mathrm{~h}$. Table S10. Regions with $\Delta \% \mathrm{D}_{\mathrm{av}} \geq 10 \%$. Video S1. Morphing conformations between WT NQO1 $1_{\text {dic }}$ and T16M mutated model. Video S2. Morphing conformations between WT NQO1 $1_{\text {dic }}$ and WT NQO1 $1_{\text {holo }}$ states. Video S3. Morphing conformations between WT NQO1 $1_{\text {dic }}$ and Y20N mutated model. Video S4. Morphing conformations between WT NQO1 ${ }_{\text {holo }}$ and Y20N mutated model. Video S5. Morphing conformations between WT NQO1 ${ }_{\text {dic }}$ and A29T mutated model. Video S6. Morphing conformations between WT NQO1 $1_{\text {holo }}$ and A29T mutated model.

Author Contributions: Conceptualization, A.L.P.; methodology, M.C.-M., E.S. and A.L.P.; software, M.C.-M. and A.L.P.; validation, M.C.-M., E.S. and A.L.P; formal analysis, J.L.P.-G., M.C.-M., I.S.-R., E.S. and A.L.P.; investigation, J.L.P.-G., M.C.-M., I.S.-R. and A.L.P.; resources, E.S. and A.L.P.; data curation, M.C.-M., E.S. and A.L.P.; writing-original draft preparation, M.C.-M., E.S. and A.L.P.; writing-review and editing, J.L.P.-G., M.C.-M., I.S.-R., E.S. and A.L.P.; visualization, A.L.P.; supervision, A.L.P.; project administration, A.L.P.; funding acquisition, E.S. and A.L.P. All authors have read and agreed to the published version of the manuscript.

Funding: This work was funded by the ERDF/Spanish Ministry of Science, Innovation and Universities-State Research Agency (Grant RTI2018-096246-B-I00, to A.L.P., and SAF2015-69796, to E.S.) and Consejería de Economía, Conocimiento, Empresas y Universidad, Junta de Andalucía (Grant P18-RT-2413, to A.L.P.). The funding sources had no role in the design of this study, analysis of data or preparation of the manuscript.

Acknowledgments: We thank Daniela Segura-Navarro for her help in the preparation of Table S10.

Conflicts of Interest: The authors declare no conflict of interest.

\section{References}

1. Shendure, J.; Akey, J.M. The origins, determinants, and consequences of human mutations. Science 2015, 349, 1478-1483. [CrossRef]

2. Lek, M.; Karczewski, K.J.; Minikel, E.V.; Samocha, K.E.; Banks, E.; Fennell, T.; O’Donnell-Luria, A.H.; Ware, J.S.; Hill, A.J.; Cummings, B.B.; et al. Analysis of protein-coding genetic variation in 60,706 humans. Nature 2016, 536, 285-291. [CrossRef] [PubMed]

3. The ICGC/TCGA Pan-Cancer Analysis of Whole Genomes Consortium. Pan-cancer analysis of whole genomes. Nature 2020, 578, 82-93. [CrossRef] [PubMed]

4. Kiryluk, K.; Goldstein, D.B.; Rowe, J.W.; Gharavi, A.G.; Wapner, R.; Chung, W.K. Precision Medicine in Internal Medicine. Ann. Intern. Med. 2019, 170, 635-642. [CrossRef]

5. Stein, A.; Fowler, D.M.; Hartmann-Petersen, R.; Lindorff-Larsen, K. Biophysical and Mechanistic Models for Disease-Causing Protein Variants. Trends Biochem. Sci. 2019, 44, 475-488. [CrossRef]

6. Manolio, T.A.; Fowler, D.M.; Starita, L.M.; Haendel, M.A.; MacArthur, D.G.; Biesecker, L.G.; Worthey, E.; Chisholm, R.L.; Green, E.D.; Jacob, H.J.; et al. Bedside Back to Bench: Building Bridges between Basic and Clinical Genomic Research. Cell 2017, 169, 6-12. [CrossRef]

7. Casanueva, M.O.; Burga, A.; Lehner, B. Fitness trade-offs and environmentally induced mutation buffering in isogenic C. elegans. Science 2012, 335, 82-85. [CrossRef]

8. Pey, A.L.; Stricher, F.; Serrano, L.; Martinez, A. Predicted effects of missense mutations on native-state stability account for phenotypic outcome in phenylketonuria, a paradigm of misfolding diseases. Am. J. Hum. Genet. 2007, 81, 1006-1024. [CrossRef]

9. Nielsen, S.V.; Stein, A.; Dinitzen, A.B.; Papaleo, E.; Tatham, M.H.; Poulsen, E.G.; Kassem, M.M.; Rasmussen, L.J.; Lindorff-Larsen, K.; Hartmann-Petersen, R. Predicting the impact of Lynch syndrome-causing missense mutations from structural calculations. PLoS Genet. 2017, 13, e1006739. [CrossRef]

10. Abildgaard, A.B.; Stein, A.; Nielsen, S.V.; Schultz-Knudsen, K.; Papaleo, E.; Shrikhande, A.; Hoffmann, E.R.; Bernstein, I.; Gerdes, A.M.; Takahashi, M.; et al. Computational and cellular studies reveal structural destabilization and degradation of MLH1 variants in Lynch syndrome. eLife 2019, 8, e49138. [CrossRef] [PubMed] 
11. Blouin, J.M.; Bernardo-Seisdedos, G.; Sasso, E.; Esteve, J.; Ged, C.; Lalanne, M.; Sanz-Parra, A.; Urquiza, P.; de Verneuil, H.; Millet, O.; et al. Missense UROS mutations causing congenital erythropoietic porphyria reduce UROS homeostasis that can be rescued by proteasome inhibition. Hum. Mol. Genet. 2017, 26, 1565-1576. [CrossRef]

12. Boycott, K.M.; Rath, A.; Chong, J.X.; Hartley, T.; Alkuraya, F.S.; Baynam, G.; Brookes, A.J.; Brudno, M.; Carracedo, A.; den Dunnen, J.T.; et al. International Cooperation to Enable the Diagnosis of All Rare Genetic Diseases. Am. J. Hum. Genet. 2017, 100, 695-705. [CrossRef]

13. Mesa-Torres, N.; Fabelo-Rosa, I.; Riverol, D.; Yunta, C.; Albert, A.; Salido, E.; Pey, A.L. The role of protein denaturation energetics and molecular chaperones in the aggregation and mistargeting of mutants causing primary hyperoxaluria type I. PLoS ONE 2013, 8, e71963. [CrossRef]

14. Pey, A.L.; Mesa-Torres, N.; Chiarelli, L.R.; Valentini, G. Structural and Energetic Basis of Protein Kinetic Destabilization in Human Phosphoglycerate Kinase 1 Deficiency. Biochemistry 2013, 52, 1160-1170. [CrossRef]

15. Pey, A.L.; Maggi, M.; Valentini, G. Insights into human phosphoglycerate kinase 1 deficiency as a conformational disease from biochemical, biophysical, and in vitro expression analyses. J. Inherit. Metab. Dis. 2014, 37, 909-916. [CrossRef]

16. Pey, A.L.; Padin-Gonzalez, E.; Mesa-Torres, N.; Timson, D.J. The metastability of human UDP-galactose $4^{\prime}$-epimerase (GALE) is increased by variants associated with type III galactosemia but decreased by substrate and cofactor binding. Arch. Biochem. Biophys. 2014, 562, 103-114. [CrossRef]

17. Majtan, T.; Pey, A.L.; Gimenez-Mascarell, P.; Martinez-Cruz, L.A.; Szabo, C.; Kozich, V.; Kraus, J.P. Potential Pharmacological Chaperones for Cystathionine Beta-Synthase-Deficient Homocystinuria. Handb. Exp. Pharmacol. 2018, 245, 345-383.

18. Fernandez-Higuero, J.A.; Betancor-Fernandez, I.; Mesa-Torres, N.; Muga, A.; Salido, E.; Pey, A.L. Structural and functional insights on the roles of molecular chaperones in the mistargeting and aggregation phenotypes associated with primary hyperoxaluria type I. Adv. Protein Chem. Struct. Biol. 2019, 114, 119-152.

19. Medina-Carmona, E.; Betancor-Fernández, I.; Santos, J.; Mesa-Torres, N.; Grottelli, S.; Batlle, C.; Naganathan, A.N.; Oppici, O.; Cellini, B.; Ventura, S.; et al. Insight into the specificity and severity of pathogenic mechanisms associated with missense mutations through experimental and structural perturbation analyses. Hum. Mol. Genet. 2019, 28, 1-15. [CrossRef]

20. Macias, I.; Lain, A.; Bernardo-Seisdedos, G.; Gil, D.; Gonzalez, E.; Falcon-Perez, J.M.; Millet, O. Hereditary tyrosinemia type I-associated mutations in fumarylacetoacetate hydrolase reduce the enzyme stability and increase its aggregation rate. J. Biol. Chem. 2019, 294, 13051-13060. [CrossRef] [PubMed]

21. Fossbakk, A.; Kleppe, R.; Knappskog, P.M.; Martinez, A.; Haavik, J. Functional studies of tyrosine hydroxylase missense variants reveal distinct patterns of molecular defects in Dopa-responsive dystonia. Hum. Mutat. 2014, 35, 880-890. [CrossRef]

22. McCorvie, T.J.; Gleason, T.J.; Fridovich-Keil, J.L.; Timson, D.J. Misfolding of galactose 1-phosphate uridylyltransferase can result in type I galactosemia. Biochim. Biophys. Acta 2013, 1832, 1279-1293. [CrossRef]

23. McCorvie, T.J.; Kopec, J.; Pey, A.L.; Fitzpatrick, F.; Patel, D.; Chalk, R.; Streetha, L.; Yue, W.W. Molecular basis of classic galactosemia from the structure of human galactose 1-phosphate uridylyltransferase. Hum. Mol. Genet. 2016, 25, 2234-2244. [CrossRef] [PubMed]

24. Mesa-Torres, N.; Betancor-Fernández, I.; Oppici, E.; Cellini, B.; Salido, E.; Pey, A.L. Evolutionary Divergent Suppressor Mutations in Conformational Diseases. Genes 2018, 9, 352. [CrossRef]

25. Clausen, L.; Abildgaard, A.B.; Gersing, S.K.; Stein, A.; Lindorff-Larsen, K.; Hartmann-Petersen, R. Protein stability and degradation in health and disease. Adv. Protein Chem. Struct. Biol. 2019, 114, 61-83. [PubMed]

26. Scheller, R.; Stein, A.; Nielsen, S.V.; Marin, F.I.; Gerdes, A.M.; Marco, M.D.; Papaleo, E.; Lindorff-Larsen, K.; Hartmann-Petersen, R. Towards mechanistic models for genotype-phenotype correlations in phenylketonuria using protein stability calculations. Hum. Mutat. 2019, 40, 444-457. [CrossRef]

27. Erlandsen, H.; Pey, A.L.; Gamez, A.; Perez, B.; Desviat, L.R.; Aguado, C.; Koch, R.; Surendran, S.; Tyring, S.; Matalon, R.; et al. Correction of kinetic and stability defects by tetrahydrobiopterin in phenylketonuria patients with certain phenylalanine hydroxylase mutations. Proc. Natl. Acad. Sci. USA 2004, 101, 16903-16908. [CrossRef] 
28. Pey, A.L.; Majtan, T.; Sanchez-Ruiz, J.M.; Kraus, J.P. Human cystathionine beta-synthase (CBS) contains two classes of binding sites for S-adenosylmethionine (SAM): Complex regulation of CBS activity and stability by SAM. Biochem. J. 2013, 449, 109-121. [CrossRef]

29. Fortian, A.; Castano, D.; Ortega, G.; Lain, A.; Pons, M.; Millet, O. Uroporphyrinogen III synthase mutations related to congenital erythropoietic porphyria identify a key helix for protein stability. Biochemistry 2009, 48, 454-461. [CrossRef] [PubMed]

30. Schmiesing, J.; Lohmoller, B.; Schweizer, M.; Tidow, H.; Gersting, S.W.; Muntau, A.C.; Braulke, T.; Muhlhausen, C. Disease-causing mutations affecting surface residues of mitochondrial glutaryl-CoA dehydrogenase impair stability, heteromeric complex formation and mitochondria architecture. Hum. Mol. Genet. 2017, 26, 538-551. [CrossRef]

31. Naganathan, A.N. Modulation of allosteric coupling by mutations: From protein dynamics and packing to altered native ensembles and function. Curr. Opin. Struct. Biol. 2018, 54, 1-9. [CrossRef] [PubMed]

32. Adzhubei, I.A.; Schmidt, S.; Peshkin, L.; Ramensky, V.E.; Gerasimova, A.; Bork, P.; Kondrashov, A.S.; Sunyaev, S.R. A method and server for predicting damaging missense mutations. Nat. Methods 2010, 7, 248-249. [CrossRef]

33. Guerois, R.; Nielsen, J.E.; Serrano, L. Predicting changes in the stability of proteins and protein complexes: A study of more than 1000 mutations. J. Mol. Biol. 2002, 320, 369-387. [CrossRef]

34. Sanchez, I.E.; Tejero, J.; Gomez-Moreno, C.; Medina, M.; Serrano, L. Point mutations in protein globular domains: Contributions from function, stability and misfolding. J. Mol. Biol. 2006, 363, 422-432. [CrossRef]

35. Tokuriki, N.; Stricher, F.; Schymkowitz, J.; Serrano, L.; Tawfik, D.S. The stability effects of protein mutations appear to be universally distributed. J. Mol. Biol. 2007, 369, 1318-1332. [CrossRef] [PubMed]

36. Nisthal, A.; Wang, C.Y.; Ary, M.L.; Mayo, S.L. Protein stability engineering insights revealed by domain-wide comprehensive mutagenesis. Proc. Natl. Acad. Sci. USA 2019, 116, 16367-16377. [CrossRef]

37. Khan, S.; Vihinen, M. Performance of protein stability predictors. Hum. Mutat. 2010, 31, 675-684. [CrossRef]

38. Roscoe, B.P.; Thayer, K.M.; Zeldovich, K.B.; Fushman, D.; Bolon, D.N. Analyses of the effects of all ubiquitin point mutants on yeast growth rate. J. Mol. Biol. 2013, 425, 1363-1377. [CrossRef]

39. Pey, A.L. Biophysical and functional perturbation analyses at cancer-associated P187 and K240 sites of the multifunctional NADP(H):quinone oxidoreductase 1. Int. J. Biol. Macromol. 2018, 118, 1912-1923. [CrossRef] [PubMed]

40. Pey, A.L.; Megarity, C.F.; Timson, D.J. FAD binding overcomes defects in activity and stability displayed by cancer-associated variants of human NQO1. Biochim. Biophys. Acta 2014, 1842, 2163-2173. [CrossRef]

41. Medina-Carmona, E.; Palomino-Morales, R.J.; Fuchs, J.E.; Padín-Gonzalez, E.; Mesa-Torres, N.; Salido, E.; Timson, D.J.; Pey, A.L. Conformational dynamics is key to understanding loss-of-function of NQO1 cancer-associated polymorphisms and its correction by pharmacological ligands. Sci. Rep. 2016, 6, 20331. [CrossRef] [PubMed]

42. Claveria-Gimeno, R.; Velazquez-Campoy, A.; Pey, A.L. Thermodynamics of cooperative binding of FAD to human NQO1: Implications to understanding cofactor-dependent function and stability of the flavoproteome. Arch Biochem. Biophys. 2017, 636, 17-27. [CrossRef]

43. Medina-Carmona, E.; Fuchs, J.E.; Gavira, J.A.; Mesa-Torres, N.; Neira, J.L.; Salido, E.; Palomino-Morales, R.; Burgos, M.; Timson, D.J.; Pey, A.L. Enhanced vulnerability of human proteins towards disease-associated inactivation through divergent evolution. Hum. Mol. Genet. 2017, 26, 3531-3544. [CrossRef]

44. Medina-Carmona, E.; Neira, J.L.; Salido, E.; Fuchs, J.E.; Palomino-Morales, R.; Timson, D.J.; Pey, A.L. Site-to-site interdomain communication may mediate different loss-of-function mechanisms in a cancer-associated NQO1 polymorphism. Sci. Rep. 2017, 7, 44352. [CrossRef]

45. Munoz, I.G.; Morel, B.; Medina-Carmona, E.; Pey, A.L. A mechanism for cancer-associated inactivation of NQO1 due to P187S and its reactivation by the consensus mutation H80R. FEBS Lett. 2017, 591, 2826-2835. [CrossRef]

46. Li, R.; Bianchet, M.A.; Talalay, P.; Amzel, L.M. The three-dimensional structure of NAD(P)H:quinone reductase, a flavoprotein involved in cancer chemoprotection and chemotherapy: Mechanism of the two-electron reduction. Proc. Natl. Acad. Sci. USA 1995, 92, 8846-8850. [CrossRef]

47. Beaver, S.K.; Mesa-Torres, N.; Pey, A.L.; Timson, D.J. NQO1: A target for the treatment of cancer and neurological diseases, and a model to understand loss of function disease mechanisms. Biochim. Biophys. Acta Proteins Proteom. 2019, 1867, 663-676. [CrossRef] 
48. Pey, A.L.; Megarity, C.F.; Medina-Carmona, E.; Timson, D.J. Natural Small Molecules as Stabilizers and Activators of Cancer-Associated NQO1 Polymorphisms. Curr. Drug Targets 2016, 17, 1506-1514. [CrossRef] [PubMed]

49. Lienhart, W.D.; Gudipati, V.; Uhl, M.K.; Binter, A.; Pulido, S.A.; Saf, R.; Zangger, K.; Gruber, K.; Macheroux, P. Collapse of the native structure caused by a single amino acid exchange in human NAD(P)H:quinone oxidoreductase(1.). FEBS J. 2014, 281, 4691-4704. [CrossRef] [PubMed]

50. Chen, S.; Deng, P.S.; Bailey, J.M.; Swiderek, K.M. A two-domain structure for the two subunits of NAD(P)H:quinone acceptor oxidoreductase. Protein Sci. 1994, 3, 51-57. [CrossRef]

51. Faig, M.; Bianchet, M.A.; Talalay, P.; Chen, S.; Winski, S.; Ross, D.; Amzel, L.M. Structures of recombinant human and mouse $\mathrm{NAD}(\mathrm{P}) \mathrm{H}$ :quinone oxidoreductases: Species comparison and structural changes with substrate binding and release. Proc. Natl. Acad. Sci. USA 2000, 97, 3177-3182. [CrossRef]

52. Martinez-Limon, A.; Alriquet, M.; Lang, W.H.; Calloni, G.; Wittig, I.; Vabulas, R.M. Recognition of enzymes lacking bound cofactor by protein quality control. Proc. Natl. Acad. Sci. USA 2016, 113, 12156-12161. [CrossRef]

53. Salido, E.; Timson, D.J.; Betancor-Fernández, I.; Palomino-Morales, R.; Pey, A.L. Targeting HIF-1alpha Function in Cancer through the Chaperone Action of NQO1. Preprints 2020, 2020030285.

54. Anusevicius, Z.; Sarlauskas, J.; Cenas, N. Two-electron reduction of quinones by rat liver NAD(P)H:quinone oxidoreductase: Quantitative structure-activity relationships. Arch. Biochem. Biophys. 2002, 404, 254-262. [CrossRef]

55. Landi, L.; Fiorentini, D.; Galli, M.C.; Segura-Aguilar, J.; Beyer, R.E. DT-Diaphorase maintains the reduced state of ubiquinones in lipid vesicles thereby promoting their antioxidant function. Free Radic. Biol. Med. 1997, 22, 329-335. [CrossRef]

56. Siegel, D.; Bolton, E.M.; Burr, J.A.; Liebler, D.C.; Ross, D. The reduction of alpha-tocopherolquinone by human $\mathrm{NAD}(\mathrm{P}) \mathrm{H}$ : Quinone oxidoreductase: The role of alpha-tocopherolhydroquinone as a cellular antioxidant. Mol. Pharmacol. 1997, 52, 300-305. [CrossRef]

57. Siegel, D.; Gustafson, D.L.; Dehn, D.L.; Han, J.Y.; Boonchoong, P.; Berliner, L.J.; Ross, D. NAD(P)H:quinone oxidoreductase 1: Role as a superoxide scavenger. Mol. Pharmacol. 2004, 65, 1238-1247. [CrossRef]

58. Ross, D.; Siegel, D. NQO1 in protection against oxidative stress. Curr. Opin. Toxicol. 2018, 7, 67-72. [CrossRef]

59. Anoz-Carbonell, E.; Timson, D.J.; Pey, A.L.; Medina, M. The Catalytic Cycle of the Antioxidant and Cancer-Associated Human NQO1 Enzyme: Hydride Transfer, Conformational Dynamics and Functional Cooperativity. Antioxidants 2020, 9, 772. [CrossRef]

60. Timson, D.J. Dicoumarol: A Drug which Hits at Least Two Very Different Targets in Vitamin K Metabolism. Curr. Drug Targets 2017, 18, 500-510. [CrossRef] [PubMed]

61. Oh, E.T.; Kim, J.W.; Kim, J.M.; Kim, S.J.; Lee, J.S.; Hong, S.S.; Goodwin, J.; Ruthenborg, R.J.; Jung, M.G.; Lee, H.J.; et al. NQO1 inhibits proteasome-mediated degradation of HIF-1alpha. Nat. Commun. 2016, 7, 13593. [CrossRef]

62. Asher, G.; Tsvetkov, P.; Kahana, C.; Shaul, Y. A mechanism of ubiquitin-independent proteasomal degradation of the tumor suppressors p53 and p73. Genes Dev. 2005, 19, 316-321. [CrossRef] [PubMed]

63. Nolan, K.A.; Zhao, H.; Faulder, P.F.; Frenkel, A.D.; Timson, D.J.; Siegel, D.; Ross, D.; Burke, T.R., Jr.; Stratford, I.J.; Bryce, R.A. Coumarin-based inhibitors of human NAD(P)H:quinone oxidoreductase-1. Identification, structure-activity, off-target effects and in vitro human pancreatic cancer toxicity. J. Med. Chem. 2007, 50, 6316-6325. [CrossRef]

64. Cullen, J.J.; Hinkhouse, M.M.; Grady, M.; Gaut, A.W.; Liu, J.; Zhang, Y.P.; Weydert, C.J.; Domann, F.E.; Oberley, L.W. Dicumarol inhibition of NADPH:quinone oxidoreductase induces growth inhibition of pancreatic cancer via a superoxide-mediated mechanism. Cancer Res. 2003, 63, 5513-5520.

65. Lajin, B.; Alachkar, A. The NQO1 polymorphism C609T (Pro187Ser) and cancer susceptibility: A comprehensive meta-analysis. Br. J. Cancer 2013, 109, 1325-1337. [CrossRef]

66. Traver, R.D.; Horikoshi, T.; Danenberg, K.D.; Stadlbauer, T.H.; Danenberg, P.V.; Ross, D.; Gibson, N.W. $\mathrm{NAD}(\mathrm{P}) \mathrm{H}$ :quinone oxidoreductase gene expression in human colon carcinoma cells: Characterization of a mutation which modulates DT-diaphorase activity and mitomycin sensitivity. Cancer Res. 1992, 52, 797-802.

67. Traver, R.D.; Siegel, D.; Beall, H.D.; Phillips, R.M.; Gibson, N.W.; Franklin, W.A.; Ross, D. Characterization of a polymorphism in NAD(P)H: Quinone oxidoreductase (DT-diaphorase). Br. J. Cancer 1997, 75, 69-75. [CrossRef] 
68. Siegel, D.; Anwar, A.; Winski, S.L.; Kepa, J.K.; Zolman, K.L.; Ross, D. Rapid polyubiquitination and proteasomal degradation of a mutant form of NAD(P)H:quinone oxidoreductase 1. Mol. Pharmacol. 2001, 59, 263-268. [CrossRef]

69. Eguchi-Ishimae, M.; Eguchi, M.; Ishii, E.; Knight, D.; Sadakane, Y.; Isoyama, K.; Yabe, H.; Mizutani, S.; Greaves, M. The association of a distinctive allele of $\mathrm{NAD}(\mathrm{P}) \mathrm{H}$ :quinone oxidoreductase with pediatric acute lymphoblastic leukemias with MLL fusion genes in Japan. Haematologica 2005, 90, 1511-1515. [PubMed]

70. Lienhart, W.D.; Strandback, E.; Gudipati, V.; Koch, K.; Binter, A.; Uhl, M.K.; Rantasa, D.M.; Bourgeois, B.; Madl, T.; Zangger, K.; et al. Catalytic competence, structure and stability of the cancer-associated R139W variant of the human NAD(P)H:quinone oxidoreductase 1 (NQO1). FEBS J. 2017, 284, 1233-1245. [CrossRef]

71. Pan, S.S.; Forrest, G.L.; Akman, S.A.; Hu, L.T. NAD(P)H:quinone oxidoreductase expression and mitomycin C resistance developed by human colon cancer HCT 116 cells. Cancer Res. 1995, 55, 330-335. [PubMed]

72. Asher, G.; Dym, O.; Tsvetkov, P.; Adler, J.; Shaul, Y. The crystal structure of NAD(P)H quinone oxidoreductase 1 in complex with its potent inhibitor dicoumarol. Biochemistry 2006, 45, 6372-6378. [CrossRef]

73. Fuchs, J.E.; Muñoz, I.G.; Timson, D.J.; Pey, A.L. Experimental and computational evidence on conformational fluctuations as a source of catalytic defects in genetic diseases. RSC Adv. 2016, 6, 58604. [CrossRef]

74. Robertson, A.D.; Murphy, K.P. Protein Structure and the Energetics of Protein Stability. Chem. Rev. 1997, 97, 1251-1268. [CrossRef] [PubMed]

75. Leaver-Fay, A.; Tyka, M.; Lewis, S.M.; Lange, O.F.; Thompson, J.; Jacak, R.; Kaufman, K.; Renfrew, P.D.; Smith, C.A.; Sheffler, W.; et al. ROSETTA3: An object-oriented software suite for the simulation and design of macromolecules. Methods Enzymol. 2011, 487, 545-574.

76. Alford, R.F.; Leaver-Fay, A.; Jeliazkov, J.R.; O’Meara, M.J.; DiMaio, F.P.; Park, H.; Shapovalov, M.V.; Renfrew, P.D.; Mulligan, V.K.; Kappel, K.; et al. The Rosetta All-Atom Energy Function for Macromolecular Modeling and Design. J. Chem. Theory Comput. 2017, 13, 3031-3048. [CrossRef]

77. Kellogg, E.H.; Leaver-Fay, A.; Baker, D. Role of conformational sampling in computing mutation-induced changes in protein structure and stability. Proteins 2011, 79, 830-838. [CrossRef] [PubMed]

78. Krieger, E.; Vriend, G. YASARA View-Molecular graphics for all devices—From smartphones to workstations. Bioinformatics 2014, 30, 2981-2982. [CrossRef]

79. Pandurangan, A.P.; Ochoa-Montano, B.; Ascher, D.B.; Blundell, T.L. SDM: A server for predicting effects of mutations on protein stability. Nucleic Acids Res. 2017, 45, W229-W235. [CrossRef]

80. Bianchet, M.A.; Faig, M.; Amzel, L.M. Structure and mechanism of NAD[P]H:quinone acceptor oxidoreductases (NQO). Methods Enzymol. 2004, 382, 144-174.

81. Dehouck, Y.; Kwasigroch, J.M.; Gilis, D.; Rooman, M. PoPMuSiC 2.1: A web server for the estimation of protein stability changes upon mutation and sequence optimality. BMC Bioinform. 2011, 12, 151. [CrossRef]

82. Rodrigues, C.H.; Pires, D.E.; Ascher, D.B. DynaMut: Predicting the impact of mutations on protein conformation, flexibility and stability. Nucleic Acids Res. 2018, 46, W350-W355. [CrossRef]

83. Laimer, J.; Hiebl-Flach, J.; Lengauer, D.; Lackner, P. MAESTROweb: A web server for structure-based protein stability prediction. Bioinformatics 2016, 32, 1414-1416. [CrossRef]

84. Laimer, J.; Hofer, H.; Fritz, M.; Wegenkittl, S.; Lackner, P. MAESTRO-multi agent stability prediction upon point mutations. BMC Bioinform. 2015, 16, 116. [CrossRef]

85. Parthiban, V.; Gromiha, M.M.; Schomburg, D. CUPSAT: Prediction of protein stability upon point mutations. Nucleic Acids Res. 2006, 34, W239-W242. [CrossRef]

86. Sim, N.L.; Kumar, P.; Hu, J.; Henikoff, S.; Schneider, G.; Ng, P.C. SIFT web server: Predicting effects of amino acid substitutions on proteins. Nucleic Acids Res. 2012, 40, W452-W457. [CrossRef]

87. Choi, Y.; Chan, A.P. PROVEAN web server: A tool to predict the functional effect of amino acid substitutions and indels. Bioinformatics 2015, 31, 2745-2747. [CrossRef] [PubMed]

88. Niroula, A.; Urolagin, S.; Vihinen, M. PON-P2: Prediction method for fast and reliable identification of harmful variants. PLoS ONE 2015, 10, e0117380. [CrossRef]

89. Schwarz, J.M.; Rodelsperger, C.; Schuelke, M.; Seelow, D. MutationTaster evaluates disease-causing potential of sequence alterations. Nat. Methods 2010, 7, 575-576. [CrossRef]

90. Vankova, P.; Salido, E.; Timson, D.J.; Man, P.; Pey, A.L. A dynamic core in human NQO1 controls the functional and stability effects of ligand binding and their communication across the enzyme dimer. Biomolecules 2019, 9, 728. [CrossRef] 
91. Medina-Carmona, E.; Rizzuti, B.; Martin-Escolano, R.; Pacheco-Garcia, J.L.; Mesa-Torres, N.; Neira, J.L.; Guzzi, R.; Pey, A.L. Phosphorylation compromises FAD binding and intracellular stability of wild-type and cancer-associated NQO1: Insights into flavo-proteome stability. Int. J. Biol. Macromol. 2019, 125, 1275-1288. [CrossRef]

92. Megarity, C.F.; Timson, D.J. Cancer-associated variants of human NQO1: Impacts on inhibitor binding and cooperativity. Biosci. Rep. 2019, 39, BSR20191874. [CrossRef]

93. Scott, K.A.; Barnes, J.; Whitehead, R.C.; Stratford, I.J.; Nolan, K.A. Inhibitors of NQO1: Identification of compounds more potent than dicoumarol without associated off-target effects. Biochem. Pharmacol. 2011, 81, 355-363. [CrossRef] [PubMed]

94. Betancor-Fernandez, I.; Timson, D.J.; Salido, E.; Pey, A.L. Natural (and Unnatural) Small Molecules as Pharmacological Chaperones and Inhibitors in Cancer. Handb. Exp. Pharmacol. 2018, 45, 345-383.

95. Jacob, J.; Duclohier, H.; Cafiso, D.S. The role of proline and glycine in determining the backbone flexibility of a channel-forming peptide. Biophys. J. 1999, 76, 1367-1376. [CrossRef]

96. Morgan, A.A.; Rubenstein, E. Proline: The distribution, frequency, positioning, and common functional roles of proline and polyproline sequences in the human proteome. PLoS ONE 2013, 8, e53785. [CrossRef] [PubMed]

97. Eriksson, A.E.; Baase, W.A.; Zhang, X.J.; Heinz, D.W.; Blaber, M.; Baldwin, E.P.; Matthews, B.W. Response of a protein structure to cavity-creating mutations and its relation to the hydrophobic effect. Science 1992, 255, 178-183. [CrossRef]

98. Xue, M.; Wakamoto, T.; Kejlberg, C.; Yoshimura, Y.; Nielsen, T.A.; Risor, M.W.; Sanggaard, K.W.; Kitahara, R.; Mulder, F.A.A. How internal cavities destabilize a protein. Proc. Natl. Acad. Sci. USA 2019, 116, 21031-21036. [CrossRef]

99. Pey, A.L. Towards Accurate Genotype-Phenotype Correlations in the CYP2D6 Gene. J. Pers. Med. 2020, 10, 158. [CrossRef]

100. Gersting, S.W.; Kemter, K.F.; Staudigl, M.; Messing, D.D.; Danecka, M.K.; Lagler, F.B.; Sommerhoff, C.P.; Roscher, A.A.; Muntau, A.C. Loss of function in phenylketonuria is caused by impaired molecular motions and conformational instability. Am. J. Hum. Genet. 2008, 83, 5-17. [CrossRef]

101. Rajasekaran, N.; Naganathan, A.N. A Self-Consistent Structural Perturbation Approach for Determining the Magnitude and Extent of Allosteric Coupling in Proteins. Biochem. J. 2017, 474, 2379-2388. [CrossRef]

102. Rajasekaran, N.; Sekhar, A.; Naganathan, A.N. A Universal Pattern in the Percolation and Dissipation of Protein Structural Perturbations. J. Phys. Chem. Lett. 2017, 8, 4779-4784. [CrossRef]

103. Rajasekaran, N.; Suresh, S.; Gopi, S.; Raman, K.; Naganathan, A.N. A General Mechanism for the Propagation of Mutational Effects in Proteins. Biochemistry 2017, 56, 294-305. [CrossRef]

Publisher's Note: MDPI stays neutral with regard to jurisdictional claims in published maps and institutional affiliations.

(C) 2020 by the authors. Licensee MDPI, Basel, Switzerland. This article is an open access article distributed under the terms and conditions of the Creative Commons Attribution (CC BY) license (http://creativecommons.org/licenses/by/4.0/). 